\title{
Invariant measures of homeomorphisms and applications to the stability of an hyperbolic PDE
}

\section{Mohammed Aassila}

\begin{abstract}
Using the invariant measures of homeomorphisms, we study in this paper the asymptotic behavior of the energy $E(t)$ of an hyperbolic partial differential equation in a moving domain. The behavior of $E(t)$ as $t \rightarrow \infty$ depends essentially on the number theoretical characteristics of the rotation number of the homeomorphism.
\end{abstract}

Keywords: stability, global existence, asymptotic bahaviour.

Mathematical subject classification: 35L70, 35B40.

\section{Introduction}

The study of the so-called Fermi accelerators becomes more and more extensive. The name comes from Fermi's considerations on the possible mechanism of cosmic rays acceleration [13]. In the later studies up to contemporary ones, they serve as simple prototypes of the externally driven dynamical systems, mainly in the connection with the deterministic and chaotic behavior of the classical and quantum systems. The first mechanical models were proposed by Ulam [33], the rigorous results in Newtonian mechanics, Pustyl'nikov [28, 29], and in spatialrelavistic classical mechanics, Pustyl'nikov [30, 31], were obtained much later. Only as a sample of papers in nonrelavistic quantum mechanics let us mention Karner [19], Dodonov, Klimov and Nikonov [11]. Similar problems for classical wave equation: Balasz [2], Cooper [5], Cooper and Koch [6], Gonzalez [16], Perla Menzala [27], Nakao [25], Nakao and Narazaki [26], Ferrel and Medeiros [14], Aassila [1], and the references cited therein. Maxwell equations were also considered by Cooper [7]. An analogous model in quantum field theory was treated, for example, by Moore [24], Calucci [3], Dodonov, Klimov and Nikonov 
[12], Johnston and Sarkar [18]. In the present paper, we continue and extend the study for classical d'Alembert equation.

Let us consider the one-dimensional wave equation in a domain with one spatial boundary fixed and the second one moving slower than the wave velocity. Let us assume that the boundary motion is described by a Lipschitz continuous function $s(t)$ and assume that the field satisfies either Dirichlet or Neumann boundary conditions. We describe the behavior of the energy $E$ of the field in more details and for a wider class of functions $s$ than the papers $[5,6,14$, $25,26,27]$ which treat only the case $s \in C^{k}(\mathbb{R})(k \geq 2), s$ is periodic and assumptions on the smallness of time variations of $s(t)$. In Nakao-Narazaki [26], existence and decay for solutions of the nonlinear wave equation in noncylindrical domains for the d'Alembert operator was investigated by employing the penalty method as in Lions [23]. In the work of Ferrel and Medeiros [14], the approach introduced by Komornik and Zuazua [21] was used to derive the exponential decay of energy. In [5], Cooper proved that if there are a finite number of reflected characteristics of period $T$, then all finite energy solutions converge as $t \rightarrow \infty$ to certain generalized solutions which can be described as square waves which travel back and forth, being reflected at the boundaries. These square waves do not have finite energy. Furthermore, the energy of all finite energy solutions grows without bound as $t \rightarrow \infty$. He gave examples where the energy of the solution grows exponentially, but the solution converges to zero a.e. This result can be summarized briefly in the statement that the energy growth of the solution caused by the moving boundary happens because of compression of a wave, not by amplification. These results have some bearing on the possibility of developing a Floquet theory for partial differential equations with time periodic coefficients. In the parabolic case this has been quite successful, see Chow, $\mathrm{Lu}$ and Pallet-Maret [4]. Also for the case of the linear wave equation with a time periodic potential, localized in space, it is possible to find a Floquet type of expansion, see Cooper, Perla Menzala and Strauss [8]. However, the situation for a hyperbolic equation with time periodic coefficients in the highest order terms is quite different because the characteristics may converge as $t \rightarrow \infty$. In [6], Cooper and Koch reduced the description of the spectrum to a study of the mapping of the characteristics through one period. They gave a precise description of the spectrum of the evolution operator considered in a complete range of Sobolev spaces.

The key of the results we present here is that the orbits of the characteristics of the wave equation are given by a Lipschitz homeomorphism $F$ of $\mathbb{R}$ which depends only on $s$ and becomes the lift of a homeomorphism of the circle when 
$s$ is periodic. According to the arithmetic properties of the rotation number of $F$ and to the regularity of $s, E$ will behave differently.

\subsection{Definitions and preliminaries}

Let $s$ be a strictly positive real function to be precised later. The problems we are considering are

$$
\begin{gathered}
u_{t t}-u_{x x}=0 \quad \text { in } \quad(0, s(t)) \times \mathbb{R}, \\
\alpha(t) u_{x}(0, t)+\beta(t) u_{t}((0, t)=0, \quad t \in \mathbb{R}, \\
u(x, 0)=u_{0}(x), \quad u_{t}(x, 0)=u_{1}(x), \quad x \in(0, s(0)),
\end{gathered}
$$

and the Dirichlet condition

$$
u(s(t), t)=0, \quad t \in \mathbb{R},
$$

or the Neumann condition

$$
u_{x}(s(t), t)=0, \quad t \in \mathbb{R},
$$

or the inhomogeneous boundary conditions (1.1), (1.3) and

$$
u(0, t)=\alpha(t), \quad u(s(t), t)=\beta(t), \quad t \in \mathbb{R} .
$$

Since it will not play a role in the mathematical analysis, the wave velocity of the field $u$ is normalized to 1 . In addition, if $s$ is periodic, then by a rescaling in the parameters, one can also take the period equal to 1 ; this will simplify our notations. The energy of the field $u$ is given by the standard expression

$$
E(t):=\frac{1}{2} \int_{0}^{s(t)}\left(\left|u_{t}(x, t)\right|^{2}+\left|u_{x}(x, t)\right|^{2}\right) d x, \quad t \in \mathbb{R} .
$$

The aim of this paper is to study the asymptotic behavior as $t \rightarrow \infty$ of $E(t)$. Section 2 is devoted to some preliminary results which will be needed later. In section 3 we give an explicit and detailed spectral analysis. In section 4, we study problem (1.1)-(1.3) with boundary conditions (1.4) and (1.5). According to an explicit relation between $\alpha, \beta$ and $F^{\prime}$ at the periodic point, the energy may grow exponentially, tend to zero exponentially, or remain bounded. In section 5, we study in detail the behavior of $E$ under the boundary condition (1.6). Finally, in section 6 , we study a stabilization problem.

To end this section, we introduce some notations and recall some known results. Let $X$ be either the set $\mathbb{Z}$ (the integers) or $\mathbb{N}$ (the nonnegative integers) or $\mathbb{Q}$ (the 
rational numbers) or $\mathbb{R}$ (the real numbers). Then $X^{*}=X \backslash\{0\}, X_{+}:=\{x \in$ $X, x \geq 0\}$, and $X_{+}^{*}=X^{*} \cap X_{+}$. Denote by $\mathbb{T}$ the one-dimensional torus (the circle of unit length) and by $X$ either $\mathbb{T}$ or $\mathbb{R}$. Let $C^{0}(\mathbb{T})$ be the space of the continuous periodic functions on $\mathbb{R}$. For a measurable function $F: X \rightarrow \mathbb{R}$, we shall denote by $F_{\min }$ and $F_{\max }$ its essential infimum and essential supremum, respectively. Let Lip $(X)$ be the space of Lipschitz continuous functions. We shall denote the Lipschitz constant of a function $F$ by

$$
L(F):=\sup _{x, y \in X, x \neq y}\left|\frac{F(x)-F(y)}{x-y}\right| .
$$

We denote by $=(\Omega):=C_{0}^{\infty}(\Omega)$ the space of fuctions indefinitely differentiable and with compact support, $\mathcal{D}^{\prime}(\Omega)$ denotes its dual. The usual Sobolev spaces are denoted by $W^{m, p}(\Omega)$ and $H^{m}(\Omega)$ if $p=2$.

Let $\pi: \mathbb{R} \rightarrow \mathbb{T}, x \mapsto x+\mathbb{Z}$, be the canonical projection. For any continuous map $\bar{F}: \mathbb{T} \rightarrow \mathbb{T}$, the function $F$ satisfying $\bar{F} \circ \pi=\pi \circ F$ is called a lift of $\bar{F}$ to $\mathbb{R}$. Denote by $\operatorname{Diff}^{0}(\mathbb{R})$ the homeomorphisms on $\mathbb{R}$. One calls $\mathcal{D}^{0}(\mathbb{T})$ the set of lifts of the orientation-preserving homeomorphisms of $\mathbb{T}$, i.e., $\mathcal{D}^{0}(\mathbb{T})=\{F \in$ $\left.\operatorname{Diff}^{0}(\mathbb{R}), \quad F-I d \in C^{0}(\mathbb{T})\right\}$, and $F \in \mathcal{D}^{0}(\mathbb{T})$ is a Lipschitz homeomorphism if $F$ and $F^{-1}$ are Lipschitz continuous.

For any $F \in \mathcal{D}^{0}(\mathbb{T})$, the rotation number $\rho(F)$ is defined by

$$
\rho(F):=\lim _{n \rightarrow+\infty} \frac{F^{n}(x)-x}{n}, \quad x \in \mathbb{R},
$$

where $F^{n}=F \circ F \circ \cdots \circ F$ is the $n$-th iterate of $F$. In Herman [17, Prop. II.2.3, p. 20], the limit is proven to exist (it is a real number independent of $x$ ) and to be uniform with respect to $x$. If in the sequel $\rho(F)=\frac{p}{q}$ for $p \in \mathbb{Z}, q \in \mathbb{N}^{*}$, it is always assumed that $p$ and $q$ are relatively primes.

A point $x_{0}$ is said to be a periodic point of period $q \in \mathbb{N}^{*}$ of $F \in \mathcal{D}^{0}(\mathbb{T})$ if there exists $p \in \mathbb{N}$ such that $F^{q}\left(x_{0}\right)=x_{0}+p$. If $q=1, x_{0}$ is said to be a fixed point. One can show (Cf. Herman [17, Prop. II.5.3, p. 24]) that the existence of a periodic point $x_{0}$ for $F \in \mathcal{D}^{0}(\mathbb{T}), F^{q}\left(x_{0}\right)=x_{0}+p$, is equivalent to $\rho(F)=\frac{p}{q} \in \mathbb{Q}$, which means that if the rotation number is irrational then there are no periodic points.

Let $X$ be a compact metric space (for instance, $X=\mathbb{T}$ ) and $F: X \rightarrow X$ to be a continuous map. The measure $\mu$ is said to be an invariant measure of $F$ if and only if $\mu$ belongs to the set of probability measures on $X$ (i.e. $\mu \in\left(C^{0}(x)\right)^{\prime}$ the dual space of $C^{0}(X), \mu \geq 0$ and $\mu(X)=1$ ) and for every $\mu$-measurable set $A, \mu\left(F^{-1}(A)\right)=\mu(A)$. According to Katok and Hasselblatt [20, Th. 4.1.1], 
for any continuous map of $X$ there exists at least one invariant measure. For the particular case of $F \in \mathcal{D}^{0}(\mathbb{T})$ : if $\rho(F) \in \mathbb{R} \backslash \mathbb{Q}$, the invariant measure is in general not unique and it may be atomic, however the invariant measure of $\bar{F}:=\pi(F)$ is unique (we say that $F$ is uniquely ergodic, Cf. Herman [17, prop. II.8.5, p. 28]).

The point $x_{0}$ is said to be attracting if there exists a neighborhood $U$ of $x_{0}$ such that for all $x \in U, F^{n q}(x)-n p$ tends to $x_{0}$ as $n$ tends to $+\infty$. If $x_{0}$ is an attracting periodic point of $F^{-1}$, then $x_{0}$ is called a repelling periodic point of $F$.

\section{Preliminaries}

The sets $S_{n}$ and $\mathcal{F}_{n}$ defined by

$$
\begin{gathered}
S_{n}:=\left\{s \in C^{n}(\mathbb{T}) ; s>0, s \in \operatorname{Lip}(\mathbb{T}), L(s) \in[0,1)\right\} \\
\mathcal{F}_{n}:=\left\{F \in \mathcal{D}^{n}(\mathbb{T}) ; \quad F>I d, F, F^{-1} \in \operatorname{Lip}(\mathbb{R})\right\}
\end{gathered}
$$

and equipped with $C^{n}$-topologies are homeomorphic for any $n \in \mathbb{N} \cup\{\infty, \omega\}$. Furthermore, if $n \geq 1$, then they are open subsets of $C^{n}(\mathbb{T})$ and $\mathcal{D}^{n}(\mathbb{T})$ respectively.

Let $I d$ be the identity on $\mathbb{R}$ and $s \in \operatorname{Lip}(\mathbb{T}), L(s) \in[0,1)$. Define $h:=I d-s$ and $k:=I d+s$ on $\mathbb{R}$, then we have

Proposition 2.1. $h, k, h^{-1}, k^{-1}, k \circ h^{-1}, h \circ k^{-1}$ are Lipschitzian homeomorphisms, non-decreasing from $\mathbb{R} \rightarrow \mathbb{R}$, and

$$
\begin{aligned}
L(k) & \leq 1+L(s), & L(h) \leq 1+L(s) \\
L\left(h^{-1}\right) & \leq \frac{1}{1-L(s)}, & L\left(k^{-1}\right) \leq \frac{1}{1-L(s)} ; \\
L\left(k \circ h^{-1}\right) & \leq \frac{1+L(s)}{1-L(s)}, & L\left(h \circ k^{-1}\right) \leq \frac{1+L(s)}{1-L(s)} .
\end{aligned}
$$

Furthermore if $s$ is 1-periodic then $h, k, k \circ h^{-1}$ and $h \circ k^{-1}$ belong to $\mathcal{D}^{0}(\mathbb{T})$.

$$
h \circ k^{-1}<I d<k \circ h^{-1} .
$$

Finally, let $F \in \mathcal{F}_{0}$, then there exists $s \in S_{0}$ such that

$$
F=(I d+s) \circ(I d-s)^{-1}=I d+2 s \circ(I d-s)^{-1}
$$

and

$$
s=\left(\frac{F-I d}{2}\right) \circ\left(\frac{F+I d}{2}\right)^{-1} .
$$


In addition, $L(s) \leq \frac{L(F)-1}{L(F)+1}$. If $F \in \mathcal{D}^{0}(\mathbb{T})$, then $s \in C^{0}(\mathbb{T})$.

Proof. Since $s \in C^{0}(\mathbb{R})$, then $h \in C^{0}(\mathbb{R}) ; s \in \operatorname{Lip}(\mathbb{R}), L(s) \in[0,1)$, thus $0<1-L(s) \leq h^{\prime} \leq 1+L(s)$ a.e. Consequently $h: \mathbb{R} \rightarrow \mathbb{R}$ is strictly increasing and hence is injective. Since

$$
\lim _{t \rightarrow+\infty} h(t)=+\infty \text { and } \lim _{t \rightarrow-\infty} h(t)=-\infty,
$$

we deduce that $h(\mathbb{R})=\mathbb{R}$. Whence $h: \mathbb{R} \rightarrow \mathbb{R}$ is an homeomorphism. Now, $D h^{-1}=\frac{1}{1-s^{\prime} \circ h^{-1}}$ a.e., and hence

$$
0<\frac{1}{1+L(s)} \leq D h^{-1} \leq \frac{1}{1-L(s)} \quad \text { a.e. }
$$

The same results hold for $k$.

The functions $k \circ h^{-1}$ and $h \circ k^{-1}$ are well defined, non-decreasing and are Lipschitzian homemorphisms. As

$$
D\left(k \circ h^{-1}\right)=\frac{1+s^{\prime} \circ h^{-1}}{1-s^{\prime} \circ h^{-1}} \text { a.e. we have } \quad 0<\frac{1+L(s)}{1-L(s)} \leq D\left(k \circ h^{-1}\right) \text { a.e. }
$$

The same result holds for $h \circ k^{-1}$.

Now, let us assume that $s$ is 1-periodic, by defintion $h-I d=-s$ and $k-I d=$ $s$, hence $h, k \in \mathcal{D}^{0}(\mathbb{T})$. Consequently $k \circ h^{-1}$ and $h \circ k^{-1}$ belong to $\mathcal{D}^{0}(\mathbb{T})$.

Let $F \in \mathcal{F}_{0}$, we have $L(F), L\left(F^{-1}\right) \geq 1$ since $F>I d$. Define $t:=$ $\frac{1}{2}(F+I d)$, then $t: \mathbb{R} \rightarrow \mathbb{R}$ is a Lipschitzian non-decreasing homeomorphism and $L(t)=\frac{L(F)+1}{2}$. Let $s:=\frac{1}{2}(F-I d) \circ t^{-1}$, since $F>I d$, the function $s$ is well defined on $\mathbb{R}$, continue and nonnegative. Furthermore $s=\frac{1}{2}(F+I d-$ $2 I d) \circ t^{-1}=I d-t^{-1}$ and hence $(I d+s) \circ(I d-s)^{-1}=F$.

It follows easily that

$$
\begin{aligned}
F=(I d+s) \circ(I d-s)^{-1} & =(I d-s+2 s) \circ(I d-s)^{-1} \\
& =I d+2 s \circ(I d-s)^{-1}
\end{aligned}
$$

and on the other hand

$$
s=\frac{1}{2}(F-I d) \circ t^{-1}=\left(\frac{F-I d}{2}\right) \circ\left(\frac{F+I d}{2}\right)^{-1} .
$$


Consequently, $s \in \operatorname{Lip}(\mathbb{R})$; and since

$$
s^{\prime}=\frac{F^{\prime} \circ t^{-1}-1}{F^{\prime} \circ t^{-1}+1} \text { a.e., we have } L(s) \leq \frac{L(F)-1}{L(F)+1} .
$$

If $F \in \mathcal{D}^{0}(\mathbb{T})$, then $F(x+1)=F(x)+1$ for all $x \in \mathbb{R}$ and we have $s(x+1)=$ $s(x)$ for all $x \in \mathbb{R}$.

Proposition 2.2. Let $\Sigma \subset \mathbb{R}^{2}$ be a nonempty open and connected domain such that for all $y \in \mathbb{R}, J^{y}:=\{x \in \mathbb{R} ;(x, y) \in \Sigma\}$ and for all $x \in \mathbb{R}, J_{x}:=\{y \in$ $\mathbb{R} ;(x, y) \in \Sigma\}$ are intervals of $\mathbb{R}$. Let $K_{1}:=\{x \in \mathbb{R} ; \Sigma \cap(\{x\} \times \mathbb{R}) \neq \emptyset\}$ and $K_{2}:=\{y \in \mathbb{R} ; \quad \Sigma \cap(\mathbb{R} \times\{y\}) \neq \emptyset\}$. Then, $K_{1}$ and $K_{2}$ are nonempty open intervals in $\mathbb{R}$. Let $\phi \in H_{l o c}^{1}(\Sigma)$ be such that

$$
\phi_{x y}=0 \quad \text { in } \mathcal{D}^{\prime}(\Sigma)
$$

Then there exist two functions $f \in H_{l o c}^{1}\left(K_{1}\right)$ and $g \in H_{l o c}^{1}\left(K_{2}\right)$ such that

$$
\phi(x, y)=f(x)+g(y) \text { a.e. in } \Sigma .
$$

Proof. It is evident that $K_{1}$ and $K_{2}$ are open intervals.

Clearly, $\phi \in H_{l o c}^{1}(\Sigma)$ implies that $\phi_{x} \in L_{l o c}^{2}(\Sigma) \subset L_{l o c}^{1}(\Sigma)$ and $\phi_{x y}=0$ in $\mathcal{D}^{\prime}(\Sigma)$ implies that $\partial_{y}\left(\phi_{x}\right) \in L^{1}(\Sigma)$. Thanks to [22, Th. 5.6.3] there exists a function $u \in L_{l o c}^{1}(\Sigma)$ such that:

(i) $u=\phi_{x}$ a.e. in $\Sigma$;

(ii) there exists $\widehat{K}_{1} \subset K_{1}$ such that $m\left(K_{1} \backslash \widehat{K}_{1}\right)=0$ and for all $x \in \widehat{K}_{1}$, the application $J_{x} \ni y \mapsto u(x, y)$ is absolutely continuous; $m$ is the Lebesgue measure;

(iii) $u_{y}=\phi_{x y}$ a.e. in $\Sigma$.

By Fubini's theorem: (iii) $\Rightarrow$ (iv): there exists $\widetilde{K}_{1} \subset K_{1}$ such that $m\left(K_{1} \backslash\right.$ $\left.\widetilde{K}_{1}\right)=0$ and for all $x \in \widetilde{K}_{1}, u_{y}(x, y)=0$ for almost all $y \in J_{x}$.

Let $K_{1}^{\prime}:=\widehat{K}_{1} \cap \widetilde{K}_{1} ; m\left(K_{1} \backslash K_{1}^{\prime}\right)=0$. Assumptions (ii) and (iv) imply that for all $x \in K_{1}^{\prime}$, the application $J_{x} \ni y \mapsto u(x, y)$ is constant.

Let $\varphi: K_{1} \rightarrow \mathbb{R}$ the function defined by $\varphi(x):=u(x, y)$ if $x \in K_{1}^{\prime}$ and $y \in J_{x}$ ( $\varphi$ is arbitrary on $K_{1} \backslash K_{1}^{\prime}$ which is of Lebesgue measure equal to zero). Let us prove that $\varphi \in L_{l o c}^{2}\left(K_{1}\right)$. It is sufficient to prove that for all $x \in K_{1}$, there exists 
$r>0$ such that $\varphi \in L^{2}((x-r, x+r))$. Let $x_{0} \in K_{1}$, then $J_{x_{0}} \neq \emptyset$ and there exists $r>0$ such that $B^{\infty}\left(\left(x_{0}, y_{0}\right), r\right) \subset \Sigma$ for certain element $y_{0} \in J_{x}$, with

$$
B^{\infty}\left(\left(x_{0}, y_{0}\right), r\right):=\left\{(x, y) \in \mathbb{R}^{2} ; \max \left\{\left|x-x_{0}\right|,\left|y-y_{0}\right|\right\}<r\right\} .
$$

Hence, for all $x \in\left(x_{0}-r, x_{0}+r\right) \cap K_{1}^{\prime}$ and for all $y \in\left(y_{0}-r, y_{0}+r\right)$, we have $u(x, y)=\varphi(x)$. Assumption (i) implies that for almost all $y \in\left(y_{0}-r, y_{0}+r\right)$, we have $u(\cdot, y) \in L^{2}\left(\left(x_{0}-r, x_{0}+r\right)\right)$. Let $y_{1} \in\left(y_{0}-r, y_{0}+r\right)$ satisfies this relation, then for all $x \in\left(x_{0}-r, x_{0}+r\right) \cap K_{1}^{\prime}$, we have $\varphi(x)=u\left(x, y_{1}\right)$ and hence $\varphi \in L^{2}\left(\left(x_{0}-r, x_{0}+r\right)\right)$.

Since $\phi, \phi_{x} \in L_{l o c}^{1}(\Sigma)$, we use for the second time [22, Th. 5.6.3], and hence there exists $v \in L_{l o c}^{1}(\Sigma)$ such that:

(i') $v=\phi$ a.e. in $\Sigma$;

(ii') there exists $\widehat{K}_{2} \subset K_{2}$ such that $m\left(K_{2} \backslash \widehat{K}_{2}\right)=0$ and for all $y \in \widehat{K}_{2}$, the application $J_{y} \ni x \mapsto v(x, y)$ is absolutely continuous;

(iii') $v_{x}=\phi_{x}=u$ a.e. in $\Sigma$.

By Fubini's theorem: (iii') $\Rightarrow$ (iv'): there exists $\widetilde{K}_{2} \subset K_{2}$ such that $m\left(K_{2} \backslash\right.$ $\left.\widetilde{K}_{2}\right)=0$ and for all $x \in \widetilde{K}_{2}, v_{x}(x, y)=u(x, y)$ for almost all $x \in J_{y}$.

By (ii'), $a_{y} \in J^{y}$ being arbitrary

$$
\forall y \in \widehat{K}_{2}, \quad \forall x \in J^{y}, \quad v(x, y)=v\left(a_{y}, y\right)+\int_{a_{y}}^{x} v_{x}(z, y) d z .
$$

Thanks to (iv'), we deduce from (2.1) that

$$
\begin{aligned}
\forall y \in \widehat{K}_{2} \cap \widetilde{K}_{2}=: K_{2}^{\prime}, \forall x \in J^{y}, \quad v(x, y) & =v\left(a_{y}, y\right)+\int_{a_{y}}^{x} u(z, y) d z \\
& =v\left(a_{y}, y\right)+\int_{a_{y}}^{x} \varphi(z) d z .
\end{aligned}
$$

Let $a_{0} \in K_{1}$, we define

$$
\begin{aligned}
& \forall x \in K_{1}, \quad f(x):=\int_{a_{0}}^{x} \varphi(z) d z, \\
& \forall y \in K_{2}, \quad g(y):=v\left(a_{y}, y\right)+\int_{a_{y}}^{a_{0}} \varphi(z) d z .
\end{aligned}
$$


Since $\varphi \in L_{l o c}^{1}\left(K_{1}\right), f$ is absolutely continuous on $K_{1}$, and $f^{\prime}=\varphi$ a.e. on $K_{1}$. Hence $f \in H_{l o c}^{1}\left(K_{1}\right)$. On the other hand, we know that $g: K_{2} \rightarrow \mathbb{R}$ is a function and thus

$$
\phi(x, y)=v(x, y)=f(x)+g(y) \quad \text { a.e. in } \quad \Sigma .
$$

Similarly, by exchanging the roles of $f$ and $g$, there exist functions $g_{1} \in H_{l o c}^{1}\left(K_{2}\right)$ and $f_{1}: K_{1} \rightarrow \mathbb{R}$ such that $\phi(x, y)=f_{1}(x)+g_{1}(y)$ a.e. in $\Sigma$. Hence $f_{1}=f-c$ and $g_{1}=g+c$ a.e. for a certain constant $c \in \mathbb{R}$. Consequently

$$
\phi(x, y)=f(x)+g(y) \quad \text { a.e. in } \Sigma,
$$

with $f \in H_{l o c}^{1}\left(K_{1}\right)$ and $g \in H_{l o c}^{1}\left(K_{2}\right)$.

Proposition 2.3. Let $J:=\left[a_{1}, a_{2}\right] \subset \mathbb{R}, a_{1}<a_{2}, F: J \rightarrow J$ a $C^{1}$-class increasing function such that:

(a) $a_{1}$ is a fixed point of $F$ and $F^{\prime}\left(a_{1}\right)<1$,

(b) $a_{2}$ is a fixed point of $F$ and $F^{\prime}\left(a_{2}\right) \geq 1$,

(c) $\forall x \in\left(a_{1}, a_{2}\right), F(x)<x$.

Let $G \in \operatorname{Lip}(J)$ be such that $G>0$. Let $f \in L^{2}(J),\|f\|_{J}:=\|f\|_{L^{2}(J)}>0$, and $l: J \rightarrow \mathbb{R}_{+}, x \mapsto \prod_{k=0}^{+\infty} \frac{G\left(a_{1}\right)}{G \circ F^{k}(x)}$. Then, $l$ is well-posed and continuous on $\left[a_{1}, a_{2}\right)$. If $G\left(a_{1}\right)<G\left(a_{2}\right)$, we set $L:=\|\sqrt{l} f\|_{J}^{2}$ :

$$
L \in \mathbb{R}_{+}^{*} \text { and }\left\|\frac{f}{\sqrt{\prod_{k=0}^{n-1} G \circ F^{k}}}\right\|_{J}^{2}=\frac{\|\sqrt{l} f\|_{J}^{2}}{G\left(a_{1}\right)^{n}}(1+o(1)) \text { if } n \rightarrow+\infty
$$

and if $G\left(a_{1}\right)>G\left(a_{2}\right)$, we set $L^{\prime}:=\left\|\sqrt{l^{-1}} f\right\|_{J}^{2}$ :

$$
L^{\prime} \in \mathbb{R}_{+}^{*} \text { and }\left\|f \sqrt{\prod_{k=0}^{n-1} G \circ F^{k}}\right\|_{J}^{2}=L^{\prime} G\left(a_{1}\right)^{n}(1+o(1)) \text { if } n \rightarrow+\infty .
$$

Furthermore, we assume that $F^{\prime}\left(a_{2}\right)>1$ and that $f$ is $L^{\infty}$ in a neighborhood of $a_{2}$. Then, if $G\left(a_{1}\right)<G\left(a_{2}\right)$,

$$
\left\|\frac{f}{\sqrt{\prod_{k=0}^{n-1} G \circ F^{k}}}\right\|_{J}^{2}=\frac{\|\sqrt{l} f\|_{J}^{2}}{G\left(a_{1}\right)^{n}}\left(1+\mathcal{O}\left(\frac{1}{n}\right)\right) \text { if } n \rightarrow+\infty
$$


and if $G\left(a_{1}\right)>G\left(a_{2}\right)$,

$$
\left\|f \sqrt{\prod_{k=0}^{n-1} G \circ F^{k}}\right\|_{J}^{2}=\left\|\sqrt{l^{-1}} f\right\|_{J}^{2} G\left(a_{1}\right)^{n}\left(1+\mathcal{O}\left(\frac{1}{n}\right)\right) \text { if } n \rightarrow+\infty .
$$

Proof. We will prove (2.2) and (2.4). The proofs of (2.3) and (2.5) are similar. For all $n \in \mathbb{N}^{*}$ we define

$$
\begin{gathered}
K_{n}:=\left\|\frac{f}{\sqrt{\prod_{k=0}^{n-1} G \circ F^{k}}}\right\|_{J}^{2}, \quad \widehat{K}_{n}:=\frac{1}{G\left(a_{1}\right)^{n}}, \\
r_{n}:=\frac{K_{n}}{\widehat{K}_{n}}, \quad l_{n}(x):=\prod_{k=0}^{n-1} \frac{G\left(a_{1}\right)}{G \circ F^{k}(x)}>0 .
\end{gathered}
$$

We have $r_{n}=\left\|\sqrt{l_{n}} f\right\|_{J}^{2}$. We will prove that $l_{n}(x)$ converges to $l(x)$ and it is uniformly bounded, hence by the dominated convergence theorem we conclude that $\lim _{n \rightarrow+\infty} r_{n}=L$. Thus, $K_{n}=\widehat{K}_{n}(L+o(1))$ as $n \rightarrow+\infty$. Finally we prove that $\frac{K_{n}}{\widehat{K}_{n}}-L=\mathcal{O}\left(\frac{1}{n}\right)$.

For all $x \in\left[a_{1}, a_{2}\right], l_{n}(x)=\prod_{k=0}^{n-1}\left(1+v_{n}(x)\right)$ with $v_{n}(x):=\frac{G\left(a_{1}\right)-G \circ F^{k}(x)}{G \circ F^{k}(x)}>$ -1 . By the continuity of $F^{\prime}$, there exists $J_{-}:=\left[a_{1}, a_{1}+\delta_{-}\right], \delta_{-}>0$, such that for all $x \in J_{-}, F^{\prime}(x)<1$ and for all $x \in\left[a_{1}, a_{2}\right)$ there exists $n_{0}(x) \in \mathbb{N}$ such that for all $n \geq n_{0}(x), F^{n}(x) \in J_{-}$. We have

$$
\begin{aligned}
\left|v_{k}(x)\right| & =\frac{\left|\int_{a_{1}}^{F^{k}(x)} G^{\prime}(y) d y\right|}{G \circ F^{k}(x)} \\
& \leq\left|a_{1}-F^{k}(x)\right| \cdot\left\|G^{\prime}\right\|_{L^{\infty}(J)} \cdot\left\|\frac{1}{G}\right\|_{L^{\infty}(J)},
\end{aligned}
$$

and

$$
\begin{aligned}
\forall k \geq n_{0}(x), \quad\left|a_{1}-F^{k}(x)\right| & =\left|F^{k-n_{0}(x)} \circ F^{n_{0}(x)}\left(a_{1}\right)-F^{k-n_{0}(x)} \circ F^{n_{0}(x)}(x)\right| \\
& \leq\left|a_{1}-x\right| \cdot\left\|F^{\prime}\right\|_{L^{\infty}(J)}^{n_{0}(x)} \cdot\left\|F^{\prime}\right\|_{L^{\infty}\left(J_{-}\right)}^{k-n_{0}(x)} .
\end{aligned}
$$

Hence $\sum_{k=0}^{+\infty}\left|v_{k}(x)\right|<+\infty$, and consequently $\prod_{k=0}^{+\infty}\left(1+v_{k}(x)\right)$ is absolutely convergent and $l(x)$ is the pointwise limit of $l_{n}(x)\left(l>0\right.$ on $\left.\left[a_{1}, a_{2}\right)\right)$. The sequence $l_{n}$ is convergent on every compact subset of $\left[a_{1}, a_{2}\right)$, hence $l$ is continuous 
on $\left[a_{1}, a_{2}\right)$. Remark that $l\left(a_{1}\right)=1$ and $l\left(a_{2}\right)=0$ if $G\left(a_{1}\right)<G\left(a_{2}\right), l\left(a_{2}\right)=$ $+\infty$ if $G\left(a_{1}\right)>G\left(a_{2}\right)$.

Let us now prove that $l_{n}(x)$ is uniformly bounded. Assume that $G\left(a_{1}\right)<G\left(a_{2}\right)$ and let $J_{+}:=\left[a_{2}-\delta_{+}, a_{2}\right]$ where $\delta_{+}$is sufficiently small so that $\min _{J_{+}} G \geq$ $G\left(a_{1}\right)$. If $x \in J_{+} \backslash\left\{a_{2}\right\}$, we denote by $n_{x} \in \mathbb{N}$ the integer such that

$$
\begin{aligned}
& n<n_{x} \Longrightarrow F^{n}(x) \in J_{+}, \\
& n \geq n_{x} \Longrightarrow F^{n}(x) \notin J_{+} .
\end{aligned}
$$

We know that for all $k<n_{x}, \frac{G\left(a_{1}\right)}{G \circ F^{k}(x)} \leq 1$, and hence for all $x \in\left[a_{2}-\delta_{+}, a_{2}\right)$ and for all $n \leq n_{x}$ we have $l_{n}(x) \leq 1$.

If $J_{+}=J$, then $n_{x}=+\infty$, and hence for all $x \in J$, for all $n \in \mathbb{N}$, we have $0 \leq l_{n}(x) \leq 1$.

If $J_{+} \neq J$, then $n_{x}<+\infty$ and for all $x \in J_{+} \backslash\left\{a_{2}\right\}$, for all $n>n_{x}$ we have

$$
\begin{aligned}
l_{n}(x) & =l_{n-n_{x}}\left(F^{n_{x}}(x)\right) l_{n_{x}}(x) \\
& \leq l_{n-n_{x}}\left(F^{n_{x}}(x)\right) \\
& \leq \sup _{n \in \mathbb{N}} \sup _{y \notin J_{+}} l_{n}(y)=: M<+\infty .
\end{aligned}
$$

Whence, by the Lebesgue convergence theorem we get

$$
\lim _{n \rightarrow+\infty} r_{n}=\|\sqrt{l} f\|_{J}^{2}=: L>0 .
$$

If $G\left(a_{1}\right)>G\left(a_{2}\right)$, then we consider $l_{n}^{-1}$ instead of $l_{n}$.

Finally, let us prove now that $\frac{K_{n}}{K_{n}}-L=\mathcal{O}\left(\frac{1}{n}\right)$.

We assume that $F^{\prime}\left(a_{2}\right)>1$ and $f^{\prime}$ is $L^{\infty}$ in a neighborhood of $a_{2}$. For $\epsilon \in\left(0, a_{2}-a_{1}\right)$, we have

$$
\begin{aligned}
\left|\frac{K_{n}}{\widehat{K}_{n}}-L\right| & \leq \int_{J}\left|l(x)-l_{n}(x)\right| \cdot|f(x)|^{2} d x \\
& =\int_{J} l_{n}(x)\left|\frac{l(x)}{l_{n}(x)}-1\right||f(x)|^{2} d x \\
& =\int_{J} l_{n}(x)\left|\exp \left(\sum_{k=n}^{+\infty} \ln \left(1+v_{k}(x)\right)\right)-1\right||f(x)|^{2} d x .
\end{aligned}
$$

Now we have

$$
\left|\frac{K_{n}}{\widehat{K}_{n}}-L\right| \leq \int_{J} l_{n}(x) \exp \left(\sum_{k=n}^{+\infty}\left|v_{k}(x)\right|\right)\left|\sum_{k=n}^{+\infty} \ln \left(1+v_{k}(x)\right)\right||f(x)|^{2} d x,
$$


since $\left|e^{X}-1\right| \leq e^{X_{0}}|X|$ if $X \leq X_{0}$ and for all $X_{0} \geq 0$.

If we denote by

$$
\begin{gathered}
\gamma_{-}:=\left\|F^{\prime}\right\|_{L^{\infty}\left(J_{-}\right)}, \quad c_{1}:=\left\|G^{\prime}\right\|_{L^{\infty}(J)} \cdot\left\|\frac{1}{G}\right\|_{L^{\infty}(J)}, \\
\gamma_{+}:=\left\|F^{\prime}\right\|_{L^{\infty}(J)}, \quad \gamma:=\frac{\gamma_{+}}{\gamma_{-}}>1
\end{gathered}
$$

and

$$
n_{\epsilon}:=\inf \left\{n \in \mathbb{N} ; \quad F^{n}\left(a_{2}-\epsilon\right) \in J_{-}\right\}
$$

the minimal number of iterations needed to reach $J_{-}$from $a_{2}-\epsilon$. Then, we have

$$
\forall x \in\left[a_{1}, a_{2}-\epsilon\right], \quad\left|v_{k}(x)\right| \leq c_{1} \gamma^{n_{\epsilon}} \gamma_{-}^{k} .
$$

Since for all $x \in J, v_{k}(x) \geq \eta>-1$ with $\eta:=\frac{G\left(a_{1}\right)}{\|G\|_{L^{\infty}(J)}}-1<0$ we deduce that

$$
\begin{aligned}
\forall x \in[\eta, 0], \quad|\ln (1+x)| & =\frac{1}{1+\xi}|x|, \quad \text { with } \xi \in(\eta, 0) \\
& \leq \frac{1}{1+\eta}|x| .
\end{aligned}
$$

Hence,

$$
\left|\frac{K_{n}}{\widehat{K}_{n}}-L\right| \leq \frac{1}{1+\eta} \int_{J} l_{n}(x) \exp \left(\sum_{k=n}^{+\infty}\left|v_{k}(x)\right|\right)\left(\sum_{k=n}^{+\infty}\left|v_{k}(x)\right|\right)|f(x)|^{2} d x .
$$

Define

$$
\begin{gathered}
c_{2}:=\left\|\left|l-l_{n}\right| \cdot|f|^{2}\right\|_{L^{\infty}\left(a_{2}-\epsilon, a_{2}\right)}, \quad c_{3}:=\frac{c_{1}}{1-\gamma_{-}}, \quad M_{n}^{\epsilon}:=\frac{e^{c_{3} \gamma^{n} \gamma_{-}^{n}}-c_{3} \gamma^{n_{\epsilon}} \gamma_{-}^{n}}{1+\eta}, \\
N_{n}^{\epsilon}:=M_{n}^{\epsilon} M^{\prime} \int_{a_{1}}^{a_{2}}|f(x)|^{2} d x, \quad M^{\prime}:=\max (1, M) .
\end{gathered}
$$

Then, we have from (2.6)-(2.7)

$$
\begin{aligned}
\left|\frac{K_{n}}{\widehat{K}_{n}}-L\right| & \leq M_{n}^{\epsilon} \int_{a_{1}}^{a_{2}-\epsilon} l_{n}(x)|f(x)|^{2} d x+\int_{a_{2}-\epsilon}^{a_{2}}\left|l(x)-l_{n}(x)\right| \cdot|f(x)|^{2} d x \\
& \leq M_{n} \epsilon M^{\prime} \int_{a_{1}}^{a_{2}-\epsilon}|f(x)|^{2} d x+c_{2} \epsilon \\
& \leq N_{n} \epsilon+c_{2} \epsilon .
\end{aligned}
$$


Let $\tilde{d}:=\min _{x \in\left[a_{1}+\delta, a_{2}-\tilde{\delta}\right]}(x-F(x))$ where $\tilde{\delta}$ is a positive constant such that $F^{\prime} \geq$ $1+\mu>1$ on $\left[a_{2}-\tilde{\delta}, a_{2}\right]$ with $\mu \in \mathbb{R}_{+}^{*}$. Let $d:=\min _{x \in\left[a_{1}+\delta, a_{2}-\epsilon\right]}(x-F(x))>0$, $d$ depends on $\epsilon$ and tends to zero as $\epsilon$ tends to zero. For all $x \in\left[a_{1}+\delta_{-}, a_{2}-\epsilon\right]$ we have

$$
\begin{aligned}
x-F(x) & =x-a_{2}+F\left(a_{2}\right)-F(x) \\
& =\left(a_{2}-x\right)\left(F^{\prime}\left(c_{x}\right)-1\right), \quad \text { with } \quad c_{x} \in\left(x, a_{2}\right) .
\end{aligned}
$$

It is not difficult to see that $d \geq \min \{\tilde{d}, \mu \epsilon\}$ and if $\epsilon$ is small enough then $d \geq \mu \epsilon$.

We define the sequence $y_{0}:=a_{2}-\epsilon, y_{n}:=F\left(y_{n-1}\right), n \geq 1,\left(y_{n}\right)$ is a decreasing sequence which converges to $a_{1}$ and $y_{0}-y_{n} \geq n d$ if $y_{n} \geq a_{1}+\delta_{-}$. On the other hand we are looking for $n_{1}$ such that $a_{1} \leq y_{n_{1}} \leq a_{1}+\delta_{-}$and $y_{n}>a_{1}+\delta_{-}$if $n<n_{1}$. Hence $a_{1} \leq y_{n_{1}}<y_{n_{1}-1} \leq a_{2}-\epsilon-\left(n_{1}-1\right) d$ and hence $n_{\epsilon} \leq \frac{a_{2}-a_{1}}{\mu \epsilon}+1$, and then

$$
\left|\frac{K_{n}}{\widehat{K}_{n}}-L\right| \leq c_{4} \exp \left(c_{3} \gamma^{\frac{a_{2}-a_{1}}{\mu \epsilon}} \gamma_{-}^{n}\right) \gamma^{\frac{a_{2}-a_{1}}{\mu \epsilon}+1} \gamma_{-}^{n}+c_{2} \epsilon
$$

where $c_{4}:=c_{3} M^{\prime} \int_{a_{1}}^{a_{2}}|f(x)|^{2} d x$.

We impose that $\epsilon$ tends to zero when $n \rightarrow+\infty$ and that the equation

$$
c_{2} \epsilon=\gamma c_{4} \exp \left(\gamma c_{3} \gamma^{\frac{a_{2}-a_{1}}{\mu \epsilon}} \gamma_{-}^{n}\right) \gamma^{\frac{a_{2}-a_{1}}{\mu \epsilon}} \gamma_{-}^{n},
$$

is satisfied.

By taking the logarithm of the above equation and setting $c_{5}:=\log \frac{\gamma c_{4}}{c_{2}}$ we get

$$
-c_{5}+\ln \epsilon-\frac{\left(a_{2}-a_{1}\right) \ln \gamma}{\mu} \frac{1}{\epsilon}+\ln \gamma_{-}^{-1} n=\gamma c_{3} \gamma^{\frac{a_{2}-a_{1}}{\mu \epsilon}} \gamma_{-}^{n} .
$$

Since $\frac{\left(a_{2}-a_{1}\right) \ln \gamma}{\mu \epsilon}+\ln \gamma_{-} n$ cannot tend to $+\infty$ as $n$ tends to infinity, we easily deduce that for $n$ large enough

$$
\epsilon \leq \frac{c_{6}}{n}
$$

where $c_{6} \in \mathbb{R}_{+}^{*}$ is a real constant.

\section{Spectral Analysis}

Consider the operator $S f:=f \circ F$ acting on $C^{0}(\mathbb{T})$ or $L^{2}(\mathbb{T}), F$ being a diffeomorphism of the circle. Cooper and Koch [6] proved that if $F$ is a regular 
diffeomorphism of the circle $\left(F \in \mathcal{D}^{2}(\mathbb{T})\right)$, then if there exists an attracting periodic hyperbolic point then

$$
r:=\sup _{\lambda \in \sigma(S)}|\lambda|>1
$$

Otherwise, $r=1$. Hence, these spectral informations are not sufficient for the study of the energy behavior as $t \rightarrow+\infty$, especially when we dot not have an attracting periodic hyperbolic point. So, in this paper we use another method rather than spectral analysis to study the asymptotic behavior of the energy. However, in this section we present a complement to the nice work of Cooper and Koch [6].

In [6, Th. 2.6] Cooper and Koch proved the following result:

Let $F \in \operatorname{Diff}_{+}^{k}(\mathbb{T}), k \geq 2$. Let $A_{m}$ the operator in $H^{m}(\mathbb{T}), m \in \mathbb{Z}$, defined by $A_{m} f:=f \circ F^{-1}$. Let $A_{+}$(resp. $A_{-}$) the set of all the derivatives of $F$ at the attracting periodic points (resp. repelling). Let $\mu_{+}:=\inf A_{+}, \mu_{-}:=\sup A_{-}$. Let $W$ be the set of periodic points of $F$. The unique accumulation point of $A_{+}$ and $A_{-}$is assumed to be equal to 1 . The spectrum of $A_{m}$ is denoted $\sigma\left(A_{m}\right)$. Then:

Case A: $\rho(F) \in \mathbb{R} \backslash \mathbb{Q}$.

Then $\sigma\left(A_{m}\right)=\mathbb{T}$. Furthermore

$$
m \leq 0 \text { and }|\lambda|=1 \Longrightarrow \operatorname{Im} m\left(A_{m}-\lambda\right) \text { is not closed. }
$$

Case B: $\quad \rho(F) \in \mathbb{Q}$.

We assume that the Lebesgue measure of $W$ is equal to 0 if $m=0,1$, and that $W$ has its interior empty otherwise.

1. We assume that $m>0$ :

for all nonzero initial conditions $f$, the limit $\left\|A_{m}^{i} f\right\|_{H^{m}(\mathbb{T})}^{\frac{1}{|i|}}$ when $i \rightarrow+\infty$ (resp. $i \rightarrow-\infty$ ) is contained in $A_{+}^{\frac{1}{2}-m}$ (resp. $A_{-}^{m-\frac{1}{2}}$ ). Furthermore, for all $l \in A_{+}^{\frac{1}{2}-m}$ (resp. $A_{-}^{m-\frac{1}{2}}$ ), there exists $f \in H^{m}(\mathbb{T})$ such that

$$
\lim _{i \rightarrow+\infty}\left\|A_{m}^{i} f\right\|_{H^{m}(\mathbb{T})}^{\frac{1}{i \mid}}=f \quad(\text { resp. } i \rightarrow-\infty) .
$$

$\sigma\left(A_{m}\right)$ does not contain eigenvalues. There is no invariant subspaces of finite dimension. 
The closure of $\operatorname{Im} m\left(A_{m}-\lambda\right)$ is of infinite codimension if and only if $|\lambda|=1$ or $\mu_{-}^{\frac{1}{2}-m}<|\lambda|<\mu_{+}^{\frac{1}{2}-m}$.

$\operatorname{Im} m\left(A_{m}-\lambda\right)$ is dense if and only if $|\lambda| \neq 1$ and $|\lambda| \leq \mu_{-}^{\frac{1}{2}-m}$ or $|\lambda| \geq \mu_{+}^{\frac{1}{2}-m}$.

$\operatorname{Im} m\left(A_{m}-\lambda\right)$ is closed if and only if $|\lambda|$ does not belong to the closure of $\left(A_{-} \cup A_{+}\right)^{\frac{1}{2}-m}$.

2. We assume that $m \leq 0$.

For all $\lambda \in \mathbb{C}$, the closure of $\operatorname{Im} m\left(A_{m}-\lambda\right)$ is $H^{m}(\mathbb{T})$.

$N\left(A_{m}-\lambda\right)$ is of infinite dimension if and only if $\mu_{-}^{\frac{1}{2}-m}<|\lambda|<\mu_{+}^{\frac{1}{2}-m}$ or $|\lambda|=1$.

$A_{m}$ is injective if and only if $|\lambda| \neq 1$ and $|\lambda| \leq \mu_{-}^{\frac{1}{2}-m}$ or $|\lambda| \geq \mu_{+}^{\frac{1}{2}-m}$.

We present here a complement to the results of [6] with a direct and general proof. We have

Theorem 3.1. Let $X$ be a compact metric space. Let $F: X \rightarrow X$ be a continuous application, $\alpha \in C^{0}(X), \alpha \neq 0$ on $X$. Let $S f:=\alpha \cdot f \circ F$ the operator acting on $C^{0}(X)$. Then we have

$$
r:=\sup _{\lambda \in \sigma(S)}|\lambda|=\max _{\mu \in S_{F}(X)} e^{\int_{X} \ln |\alpha| d \mu},
$$

where $S_{F}(X)$ is the set of all invariant measures of $X$.

In addition, if $S$ is invertible then the spectrum of $S$ verifies:

$$
\sigma(S) \subset\left\{\lambda \in \mathbb{C} ; \min _{\mu \in S_{F}(X)} e^{\int_{X} \ln |\alpha| d \mu} \leq|\lambda| \leq \max _{\mu \in S_{F}(X)} e^{\int_{X} \ln |\alpha| d \mu}\right\} .
$$

The endpoints of the interval belong to $\sigma(S)$.

The difference between Theorem 3.1 and the results of Cooper and Koch [6] is that our results are in $C^{0}(\mathbb{T})$ or $L^{p}(\mathbb{T})$ and not simply in $L^{2}(\mathbb{T})$. A new term, $\alpha$, has been added. This has two advantages: first this permits us to obtain the spectrum in $W^{m, p}(\mathbb{T})$ (instead of $H^{m}(\mathbb{T})$ ) and to analyze various problems (see sections $4,5,6$ ). $F$ is of class $C^{1}$ (and not $C^{2}$ ), which permits us to study certain cases such as the counter-examples of Denjoy (i.e., when $F$ is not topologically conjugate to the rotation $I d+\rho(F)$ ). Finally, there is no technical conditions on the periodic points (the set $W$ in the theorem of Cooper and Koch).

In theorem 3.1 we have an unified formula for $r$, and the monotonicity and the invertibility of $F$ are not necessary. We retrieve the hypotheses of the theorem by Krylov and Bogolioubov (Cf. Queffélec [33, Th. IV.33]). 
Proof of Theorem 3.1. Let $f \in C^{0}(X)$, then for all $n \in \mathbb{N}^{*}$

$$
S^{n} f=\left(\prod_{k=0}^{n-1} \alpha \circ F^{k}\right) f \circ F^{n}
$$

and hence $\left\|S^{n}\right\|_{0}=\max _{X} \prod_{k=0}^{n-1}\left|\alpha \circ F^{k}\right|$.

Let $\mu$ be a measure of $S_{F}(X)$, then

$$
\begin{aligned}
\left\|S^{n}\right\|_{0}^{\frac{1}{n}} & =\exp \left(\max _{x \in X} \frac{1}{n} \sum_{k=0}^{n-1} \ln \left|\alpha \circ F^{k}(x)\right|\right) \\
& \geq \exp \left(\frac{1}{n} \sum_{k=0}^{n-1} \int_{X} \ln \left|\alpha \circ F^{k}\right| d \mu\right) \\
& =e^{\int_{X} \ln |\alpha| d \mu}
\end{aligned}
$$

because $\mu$ is invariant by $F$. Since $\mu$ is arbitrary and $r=\lim _{n \rightarrow+\infty}\left\|S^{n}\right\|_{0}^{\frac{1}{n}}$, then

$$
r \geq \max _{\mu \in S_{F}(X)} e^{\int_{X} \ln |\alpha| d \mu} .
$$

Let us prove that we can obtain equality as well. Let $x_{n} \in X$ be such that

$$
\frac{1}{n} \sum_{k=0}^{n-1} \ln \left|\alpha \circ F^{k}\left(x_{n}\right)\right|=\max _{x \in X} \frac{1}{n} \sum_{k=0}^{n-1} \ln \left|\alpha \circ F^{k}(x)\right|
$$

for all $n \in \mathbb{N}^{*}$. We set

$$
\Lambda_{n}:=\frac{1}{n} \sum_{k=0}^{n-1} \delta_{F^{k}\left(x_{n}\right)}, \quad \forall n \in \mathbb{N}^{*} .
$$

$\Lambda_{n}$ is a probability measure on $X$. Because of Banach-Alaoglu theorem, there exists a subsequence $\left\{\Lambda_{n_{i}}\right\}_{i \in \mathbb{N}}$ such that

$$
\lim _{i \rightarrow+\infty} \Lambda_{n_{i}}=: \Lambda \quad \text { weakly-star. }
$$

$\Lambda$ is a probability measure on $X$ invariant by $F$. Indeed,

$$
\begin{aligned}
\Lambda(f \circ F) & =\lim _{i \rightarrow+\infty} \frac{1}{n_{i}} \sum_{k=0}^{n_{i}-1} f \circ F \circ F^{k}\left(x_{n_{i}}\right) \\
& =\lim _{i \rightarrow+\infty} \frac{1}{n_{i}}\left\{\left(\sum_{k=0}^{n_{i}-1} f \circ F^{k}\left(x_{n_{i}}\right)\right)+\left(-f\left(x_{n_{i}}\right)+f \circ F^{n_{i}}\left(x_{n_{i}}\right)\right)\right\} \\
& =\Lambda f .
\end{aligned}
$$


Thanks to the Riesz representation Theorem, there exists a unique Borelian positive measure $\mu$ on $X$ such that

$$
\forall f \in C^{0}(X), \quad \Lambda f=\int_{X} f d \mu .
$$

Since $\Lambda(f \circ F)=\int_{X} f \circ F d \mu=\Lambda f=\int_{X} f d \mu$, we deduce that $\mu \in S_{F}(X)$. We choose $f:=\ln |\alpha| \in C^{0}(X)$, then since

$$
\left\|S^{n}\right\|_{0}^{\frac{1}{n}}=\exp \left(\max _{x \in X} \frac{1}{n} \sum_{k=0}^{n-1} \ln \left|\alpha \circ F^{k}(x)\right|\right)
$$

we deduce that

$$
\lim _{i \rightarrow+\infty}\left\|S^{n}\right\|_{0}^{\frac{1}{n}}=e^{\int_{X} \ln |\alpha| d \mu}
$$

with $\mu \in S_{F}(X)$. Hence $r=\max _{\mu \in S_{F}(X)} e^{\int_{X} \ln |\alpha| d \mu}$.

If, furthermore, $F$ is invertible then $S^{-1} f=\frac{1}{\alpha \circ F^{-1}} f \circ F^{-1}$, and if $r^{\prime}$ is the spectral radius of $S^{-1}$ then

$$
r^{\prime}=\max _{\mu \in S_{F}(X)} e^{\int_{X} \ln \left|\frac{1}{\alpha}\right| d \mu}=\left(\min _{\mu \in \mathcal{S}_{F}(X)} e^{\int_{X} \ln |\alpha| d \mu}\right)^{-1} .
$$

Since

$$
\inf _{\lambda \in \sigma(S)}|\lambda|=\left(\lim _{n \rightarrow+\infty}\left\|S^{-n}\right\|_{0}^{\frac{1}{n}}\right)^{-1}
$$

we deduce

$$
\sigma(S) \subset\left\{\lambda \in \mathbb{C}, \quad \frac{1}{r^{\prime}} \leq|\lambda| \leq r\right\}
$$

Corollary 3.2. Let $F \in \operatorname{Diff}_{+}^{k}(\mathbb{T}), \alpha \in C^{0}(\mathbb{T}), \alpha \neq 0$ on $\mathbb{T}$. Let $S f:=\alpha f \circ F$ the operator acting on $L^{p}(\mathbb{T}), 1 \leq p<+\infty$. Then $\sigma(S)$ is contained in the set of $\lambda \in \mathbb{C}$ such that

$$
\begin{aligned}
\min _{\mu \in S_{F}(\mathbb{T})} \exp \left(\int_{\mathbb{T}}\left(\ln |\alpha|-\frac{1}{p} \ln F^{\prime}\right) d \mu\right) & \leq|\lambda| \\
& \leq \max _{\mu \in S_{F}(\mathbb{T})} \exp \left(\int_{\mathbb{T}}\left(\ln |\alpha|-\frac{1}{p} \ln F^{\prime}\right) d \mu\right) .
\end{aligned}
$$

The endpoints of the interval belong to $\sigma(S)$. 
Proof. Consider $T f:=\beta \cdot\left(F^{\prime}\right)^{\frac{1}{p}} \cdot f \circ F, f \in L^{p}(\mathbb{T})$, with $\beta \in C^{0}(\mathbb{T})$. If $\beta=\alpha \cdot\left(F^{\prime}\right)^{\frac{-1}{p}}$, then $T=S$. But the operator $U f:=\left(F^{\prime}\right)^{\frac{1}{p}} \cdot f \circ F$ is isometric in $L^{p}(\mathbb{T})$, hence

$$
\forall n \in \mathbb{N}^{*}, \quad\left\|T^{n}\right\|_{L^{p}(\mathbb{T})}=\left\|\prod_{k=0}^{n-1} \beta \circ F^{k}\right\|_{L^{\infty}(\mathbb{T})},
$$

and as $n \rightarrow+\infty$ :

$$
\begin{aligned}
\left\|T^{n}\right\|_{L^{p}(\mathbb{T})}^{\frac{1}{n}} & =\exp \left(\frac{1}{n} \max _{x \in \mathbb{T}} \sum_{k=0}^{n-1} \ln \left|\beta \circ F^{k}(x)\right|\right) \\
& \rightarrow \max _{\mu \in S_{F}(\mathbb{T})} \exp \left(\int_{\mathbb{T}} \ln |\beta| d \mu\right)
\end{aligned}
$$

by the proof of Theorem 3.1. Since $F$ is invertible, we have

$$
T^{-1} f=\frac{1}{\beta \circ F^{-1}}\left(\frac{1}{F^{\prime} \circ F^{-1}}\right)^{\frac{1}{p}} f \circ F^{-1}
$$

and

$$
\lim _{n \rightarrow+\infty}\left\|T^{-n}\right\|_{L^{p}(\mathbb{T})}^{\frac{1}{n}}=\left(\min _{\mu \in \mathcal{S}_{F}(\mathbb{T})} e^{\int_{X} \ln |\beta| d \mu}\right)^{-1},
$$

as in Theorem 3.1.

Using the formalism of Cooper and Koch [6], we have proved the following:

Let $\bar{F} \in \operatorname{Diff}_{+}^{k}(\mathbb{T}), k \geq 1$. Let $A_{0} f:=f \circ \bar{F}$ the operator in $L^{2}(\mathbb{T})$. Then

$$
\begin{gathered}
\sigma\left(A_{0}\right) \subset \\
\left\{\lambda \in \mathbb{C} ; \min _{\mu \in \mathcal{S}_{F}(\mathbb{T})} \exp \left(\frac{-1}{2} \int_{\mathbb{T}} \ln \bar{F}^{\prime} d \mu\right) \leq|\lambda| \leq \max _{\mu \in \mathcal{S}_{F}(\mathbb{T})} \exp \left(\frac{-1}{2} \int_{\mathbb{T}} \ln \bar{F}^{\prime} d \mu\right)\right\},
\end{gathered}
$$

the endpoints of the interval belong to $\sigma\left(A_{0}\right)$. Let $A_{+}$(resp. $A_{-}$) be the set of the derivatives of $\bar{F}$ at the attracting periodic points (resp. repelling). Let $\mu_{+}:=\inf A_{+}, \mu_{-}:=\sup A_{-}$. Then:

Case A: $\rho(\bar{F}) \in \mathbb{R} \backslash \mathbb{Q}$.

Then $\sigma\left(A_{0}\right) \subset \mathbb{T}$. 
Case B: $\rho(\bar{F}) \in \mathbb{Q}$.

Then

$$
\sigma\left(A_{0}\right) \subset\left\{\lambda \in \mathbb{C} ; \sqrt{\mu_{-}} \leq|\lambda| \leq \sqrt{\mu_{+}}\right\},
$$

and the endpoints of the interval belong to $\sigma\left(A_{0}\right)$.

\section{Dissipative Boundary Conditions}

Let $s$ be a strictly positive real function to be precised later. The problem we are considering here is

$$
\begin{aligned}
& u_{t t}-u_{x x}=0 \quad \text { in } \quad(0, s(t)) \times \mathbb{R}, \\
& \alpha(t) u_{x}(0, t)+\beta(t) u_{t}(0, t)=0, \quad t \in \mathbb{R}, \\
& u(s(t), t)=0, \quad t \in \mathbb{R}, \\
& u(x, 0)=u_{0}(x), \quad u_{t}(x, 0)=u_{1}(x), \quad x \in(0, s(0)) .
\end{aligned}
$$

The energy is given by the classical formula:

$$
\forall t \in \mathbb{R}, \quad E(t):=\frac{1}{2} \int_{0}^{s(t)}\left(\left|u_{t}(x, t)\right|^{2}+\left|u_{x}(x, t)\right|^{2}\right) d x .
$$

We have the following: ( $F$ and $h$ are defined in section 2 ).

\section{Lemma 4.1.}

1. Let $s \in \operatorname{Lip}(\mathbb{R}), L(s) \in[0,1), s>0$. Then, for all $t \in \mathbb{R}$ there exists a unique $n: \mathbb{R} \rightarrow \mathbb{Z}, n(0)=0$ such that $h(t) \in I_{n(t)}:=\left[F^{n(t)}(-s(0))\right.$, $\left.F^{n(t)}(s(0))\right)$. If, furthermore, $s$ is 1-periodic, then for all $t \in \mathbb{R}^{*}$

$$
\frac{1}{\rho(F)}-\frac{s(t)}{\rho(F) t}-\frac{1+s(0)}{\rho(F)|t|}<\frac{n(t)}{t}<\frac{1}{\rho(F)}-\frac{s(t)}{\rho(F) t}+\frac{1+s(0)}{\rho(F)|t|} .
$$

2. Assume that $s \in S_{0}, \rho(F) \in \mathbb{R} \backslash \mathbb{Q}$, and let $\mu$ be the unique invariant measure of $\bar{F}$. If there exist two constants $\lambda_{1}, \lambda_{2} \in \mathbb{R}$ such that the derivative, with respect to the Lebesgue measure m, of Radon-Nikodym of $\mu$ satisfies

$$
0<\lambda_{1} \leq \frac{d \mu}{d m} \leq \lambda_{2}<+\infty
$$

then,

$$
\forall t \in \mathbb{R}, \quad \frac{\lambda_{1} E(0)}{\lambda_{2} F_{\max }^{\prime}} \leq E(t) \leq \frac{\lambda_{2} E(0)}{\lambda_{1} F_{\min }^{\prime}}
$$


Proof. 1. Thanks to Herman [17, prop. II.2.3, p. 20] we have

$$
\forall n \in \mathbb{Z}^{*}, \forall x \in \mathbb{R}, \quad-\frac{1}{n}<\frac{F^{n}(x)-x}{n}-\rho(F)<\frac{1}{n} .
$$

Choosing, $x= \pm s(0)$ and taking into account that $F^{n}(-s(0)) \leq h(t)<$ $F^{n}(s(0))$, we get

$$
\forall n \in \mathbb{Z}, \forall t \in h^{-1}\left(I_{n}\right), \quad n \rho(F)-1-s(0)<h(t)<n \rho(F)+1+s(0) .
$$

2. Let $l:=\frac{d \mu}{d m}$ and $g(x):=\int_{0}^{x} d \mu=\int_{0}^{x} l d m$. Thanks to Herman [17, p. 19] we have $g \circ F=g+\rho(F)$. It is clear that $g$ is absolutely continuous and that $\lambda_{1} \leq g^{\prime}=l \leq \lambda_{2}$ a.e. Hence $g \in \mathcal{D}^{0}(\mathbb{T})$ and $\lambda_{2}^{-1} \leq D g^{-1} \leq \lambda_{1}^{-1}$ a.e. Now, we can write $F=g^{-1} \circ R_{\rho(F)} \circ g$ where $R_{\alpha}:=I d+\alpha$. Whence, $D F^{n}=D g^{-1} \circ R_{n \rho(F)} \circ g \cdot g^{\prime}$ a.e. Thus $\lambda_{1} \lambda_{2}^{-1} \leq D F^{n} \leq \lambda_{2} \lambda_{1}^{-1}$ a.e., and consequently for all $t \in \mathbb{R}$

$$
\frac{1}{F_{\max }^{\prime}} \int_{I_{0}} \frac{\left|f^{\prime}(x)\right|^{2}}{D F^{n(t)}(x)} d x \leq E(t) \leq \frac{1}{F_{\min }^{\prime}} \int_{I_{0}} \frac{\left|f^{\prime}(x)\right|^{2}}{D F^{n(t)}(x)} d x .
$$

Now, we are in position to state and prove the main results about the asymptotic behavior of the energy associated to problem (4.1)-(4.4).

Theorem 4.2. Let $s \in \operatorname{Lip}(\mathbb{R})$ such that $L(s) \in[0,1), s>0$, and $\alpha, \beta \in$ $C^{0}(\mathbb{R})$ such that

$$
\exists v \in \mathbb{R}_{+}^{*}, \quad \forall t \in \mathbb{R}, \quad|\alpha(t) \pm \beta(t)| \geq v .
$$

Let $\left(u_{0}, u_{1}\right) \in H^{1}((0, s(0))) \times L^{2}((0, s(0)))$ be such that $u_{0}(s(0))=0$. Then, there exists a unique weak solution $u$ to problem (4.1)-(4.4) given by

$$
u(x, t)=f(t+x)+g(t-x) \quad \text { a.e. in } \Omega,
$$

$f$ and $g$ are two functions belonging to $H_{l o c}^{1}(\mathbb{R})$.

Furthermore, if $s, \alpha, \beta$ are 1-periodic and $E(0)>0$ then:

1. Assume that $\rho(F)=p$ with $p \in \mathbb{N}^{*}$ and that $F$ has only two periodic points $a_{1}, a_{2}$ in $I_{0}$, one of which $a_{1}$ is hyperbolic and attracting. Assume that $s \in C^{1}(\mathbb{T}), G:=\left|\frac{\beta-\alpha}{\beta+\alpha}\right|^{2} \cdot F^{\prime} \in \operatorname{Lip}(\mathbb{T})$ and $G\left(a_{1}\right)<G\left(a_{2}\right)$. Then,

$$
\forall t \in \mathbb{R}, \quad E(t)=\phi(t)\left\|f^{\prime}\right\|_{I_{n}(t)}^{2}
$$


with $n(t)=\frac{t}{p}+\delta_{1}(t), \delta_{1}$ bounded and $p$-periodic,

$$
\begin{aligned}
& 0<\min \left\{1, \frac{1}{F_{\text {max }}^{\prime}}\left(\frac{\beta+\alpha}{\beta-\alpha}\right)_{\text {min }}^{4}\right\} \leq \\
& \phi \leq \max \left\{1, \frac{1}{F_{\text {min }}^{\prime}}\left(\frac{\beta+\alpha}{\beta-\alpha}\right)_{\text {max }}^{4}\right\}<+\infty
\end{aligned}
$$

and

$$
\left\|f^{\prime}\right\|_{I_{n}(t)}^{2}=\frac{\left\|\sqrt{l} f^{\prime}\right\|_{I_{0}}^{2}}{G\left(a_{1}\right)^{n(t)}}(1+o(1)) \quad \text { as } \quad t \rightarrow+\infty
$$

with $l(x):=\prod_{k=0}^{+\infty} \frac{G\left(a_{1}\right)}{G \circ F^{k}(x)}, x \in I_{0} ; \quad l \in C^{0}\left(I_{0} \backslash\left\{a_{2}\right\}\right), l\left(a_{2}\right)=0$.

2. Assume that $\rho(F) \in \mathbb{R} \backslash \mathbb{Q}$ and denote by $\mu$ the unique invariant measure by $\bar{F}$. If the Radon-Nikodym derivative of $\mu$ with respect to Lebesgue's measure $m$ satisfies

$$
0<\lambda_{1} \leq \frac{d \mu}{d m} \leq \lambda_{2}<+\infty \text { for } \lambda_{1}, \lambda_{2} \in \mathbb{R}
$$

and if

$$
\int_{\mathbb{T}} \ln \left|\frac{\beta+\alpha}{\beta-\alpha}\right|^{2} d \mu=: \eta \neq 0,
$$

then for all $\epsilon>0$ there exists a real $t_{\epsilon} \geq 0$ such that for all $t \geq t_{\epsilon}$ :

$$
\begin{gathered}
\min \left\{1, \frac{1}{F_{\max }^{\prime}}\left(\frac{\beta+\alpha}{\beta-\alpha}\right)_{\min }^{4}\right\} \frac{\lambda_{1}\left\|f^{\prime}\right\|_{I_{0}}^{2}}{\lambda_{2}} \exp \left(t \frac{\eta-\epsilon}{\rho(F)}+\delta(t)(\eta-\epsilon)\right) \\
\leq E(t) \leq \\
\max \left\{1, \frac{1}{F_{\text {min }}^{\prime}}\left(\frac{\beta+\alpha}{\beta-\alpha}\right)_{\max }^{4}\right\} \frac{\lambda_{2}\left\|f^{\prime}\right\|_{I_{0}}^{2}}{\lambda_{1}} \exp \left(t \frac{\eta+\epsilon}{\rho(F)}+\delta(t)(\eta+\epsilon)\right)
\end{gathered}
$$

where $\delta$ is a bounded function in $\mathbb{R}$. 
Proof. If a weak solution $u$ exists, then thanks to proposition 2.2 it has the form

$$
u(x, t)=f(t+x)+g(t-x) \text { a.e. in } \Omega
$$

with $f, g \in H_{l o c}^{1}(\mathbb{R})$. The boundary conditions give

$$
g=-f \circ F, \quad g^{\prime}=\frac{\alpha+\beta}{\alpha-\beta} f^{\prime} \quad \text { a.e. in } \quad \mathbb{R} .
$$

Hence

$$
f^{\prime} \circ F=\frac{1}{F^{\prime}} \frac{\beta+\alpha}{\beta-\alpha} f^{\prime} \text { a.e. in } \mathbb{R} .
$$

We can then construct a solution (which is unique in fact) by successive iterations on the intervals $I_{n}$. Indeed, the initial conditions determine $f^{\prime}$ on $(0, s(0))$ a.e. and $g^{\prime}$ on $(-s(0), 0)$ a.e. By (4.5) we obtain $f^{\prime}$ on $I_{0}$, and then on $\mathbb{R}$ a.e. using (4.6). By integration, $f$ is known on $\mathbb{R}$ up to an arbitrary constant. The relation (4.5) permits us to determine $g$ on $\mathbb{R}$. We omit the details here.

Let us prove 1). We have by (4.5) for all $t \in \mathbb{R}$ :

$$
\begin{aligned}
E(t) & =\int_{h(t)}^{t}\left|g^{\prime}(x)\right|^{2} d x+\int_{t}^{k(t)}\left|f^{\prime}(x)\right|^{2} d x \\
& =\int_{h(t)}^{k(t)} \psi(x, t)\left|f^{\prime}(x)\right|^{2} d x
\end{aligned}
$$

with

$$
\psi(x, t):=\chi_{[h(t), t]}(x)\left|\frac{\beta(x)+\alpha(x)}{\beta(x)-\alpha(x)}\right|^{2}+\chi_{[t, k(t)]}(x),
$$

for all $t \in \mathbb{R}, x \in[h(t), k(t)] ; \chi_{A}$ is the characteristic function of the set $A$.

We have

$$
0<\min \left\{1,\left(\frac{\beta+\alpha}{\beta-\alpha}\right)_{\min }^{2}\right\} \leq \psi \leq \max \left\{1,\left(\frac{\beta+\alpha}{\beta-\alpha}\right)_{\max }^{2}\right\}<+\infty .
$$

We know that for all $t \in \mathbb{R}$, there exists a unique $n(t) \in \mathbb{R}$ such that $t \in h^{-1}\left(I_{n(t)}\right)$. By the variable change $y:=F^{-1}(x)$, we pass from $(h(t), k(t))$ to $I_{n(t)}$ :

$$
\begin{aligned}
\forall t \in \mathbb{R}, \quad E(t) & =\int_{h(t)}^{x_{n}(t)} \psi\left|f^{\prime}\right|^{2} d m+\int_{x_{n}(t)}^{k(t)} \psi\left|f^{\prime}\right|^{2} d m \\
& =\int_{h(t)}^{x_{n}(t)} \psi\left|f^{\prime}\right|^{2} d m+\int_{x_{n(t)-1}}^{h(t)} \psi(F(y), t)\left|f^{\prime} \circ F(y)\right|^{2} F^{\prime}(y) d y .
\end{aligned}
$$


Defining

$$
\begin{gathered}
\widetilde{\psi}(x, t):=\chi_{\left[x_{n(t)-1}, h(t)\right]}(x) \psi(F(x), t) \frac{1}{F^{\prime}(x)}\left|\frac{\beta(x)+\alpha(x)}{\beta(x)-\alpha(x)}\right|^{2} \\
+\chi_{\left[h(t), x_{n(t)]}\right.}(x) \psi(x, t)
\end{gathered}
$$

we get from (4.6) that for all $t \in \mathbb{R}, x \in I_{n(t)}$ :

$$
E(t)=\int_{I_{n}(t)} \widetilde{\psi}(x, t)\left|f^{\prime}(x, t)\right|^{2} d x .
$$

Since

$$
0<E(0) \leq \max \left\{1,\left(\frac{\beta+\alpha}{\beta-\alpha}\right)_{\max }^{2}\right\}\left\|f^{\prime}\right\|_{I_{0}}^{2},
$$

then for all $t \in \mathbb{R}$ we get

$$
\begin{aligned}
\int_{I_{n}(t)}\left|f^{\prime}(x)\right|^{2} d x & =\int_{I_{0}} \frac{\left|f^{\prime}(x)\right|^{2}}{D F^{n(t)}(x)} \prod_{k=0}^{n(t)-1}\left|\left(\frac{\beta(x)+\alpha(x)}{\beta(x)-\alpha(x)}\right) \circ F^{k}(x)\right|^{2} d x \\
& \geq\left(\frac{1}{F_{\max }^{\prime}}\left(\frac{\beta+\alpha}{\beta-\alpha}\right)_{\min }^{2}\right)^{n(t)} \frac{E(0)}{\max \left\{1,\left(\frac{\beta+\alpha}{\beta-\alpha}\right)_{\max }^{2}\right\}}>0 .
\end{aligned}
$$

For all $t \in \mathbb{R}$, we set

$$
\phi(t):=\frac{E(t)}{\int_{I_{n(t)}}\left|f^{\prime}(x)\right|^{2} d x}
$$

and hence we have

$$
\min \left\{\psi_{\min }, \frac{\psi_{\min }}{F_{\max }^{\prime}}\left(\frac{\beta+\alpha}{\beta-\alpha}\right)_{\min }^{2}\right\} \leq \phi \leq \max \left\{\psi_{\max }, \frac{\psi_{\max }}{F_{\min }^{\prime}}\left(\frac{\beta+\alpha}{\beta-\alpha}\right)_{\max }^{2}\right\},
$$
i.e.

$$
\min \left\{1, \frac{1}{F_{\max }^{\prime}}\left(\frac{\beta+\alpha}{\beta-\alpha}\right)_{\min }^{4}\right\} \leq \phi \leq \max \left\{1, \frac{1}{F_{\min }^{\prime}}\left(\frac{\beta+\alpha}{\beta-\alpha}\right)_{\max }^{4}\right\} .
$$

On the other hand, for all $n \in \mathbb{N}^{*}$, we have

$$
\int_{I_{n}}\left|f^{\prime}(x)\right|^{2} d x=\int_{I_{0}} \frac{\left|f^{\prime}(x)\right|^{2}}{\prod_{k=0}^{n-1} G \circ F^{k}(x)} d x,
$$


with $G:=F^{\prime}\left|\frac{\beta-\alpha}{\beta+\alpha}\right|^{2}$.

Thanks to proposition 2.3, and since $G \in \operatorname{Lip}(\mathbb{T}), G>0$ and $G\left(a_{1}\right)<G\left(a_{2}\right)$, we have

$$
\int_{I_{n}}\left|f^{\prime}(x)\right|^{2} d x=\frac{\left\|\sqrt{l} f^{\prime}\right\|_{I_{0}}^{2}}{G\left(a_{1}\right)^{n}}(1+o(1)) \text { as } n \rightarrow+\infty .
$$

Since $n(t)=\frac{t}{p}+\delta_{1}(t)$ where $\delta_{1}$ is a strictly increasing positive function. The proof of 1$)$ is by now complete.

2. By Lemma 4.1, we have

$$
\frac{\lambda_{1}}{\lambda_{2}} \leq D F^{n} \leq \frac{\lambda_{2}}{\lambda_{1}}
$$

On the other hand

$$
\prod_{k=0}^{n-1}\left|\left(\frac{\beta+\alpha}{\beta-\alpha}\right) \circ F^{k}(x)\right|^{2}=\exp \left(2 \sum_{k=0}^{n-1} \ln \left|\frac{\beta+\alpha}{\beta-\alpha}\right| \circ F^{k}(x)\right)
$$

and thanks to Furstenberg [15], if $u$ is a continuous and 1-periodic function then

$$
\lim _{n \rightarrow+\infty} \frac{1}{n} \sum_{k=0}^{n-1} u \circ F^{k}(x)=\int_{\mathbb{T}} u d \mu
$$

where $\mu$ is the unique invariant measure of $\bar{F}$.

Hence, if $u:=\ln \left|\frac{\beta+\alpha}{\beta-\alpha}\right|^{2}$, for all $\epsilon>0$ there exists $N_{\epsilon} \in \mathbb{N}^{*}$ such that for all $n \geq N_{\epsilon}$ and for all $x \in \mathbb{R}$ :

$$
e^{n(\eta-\epsilon)} \leq \prod_{k=0}^{n-1}\left|\frac{\beta+\alpha}{\beta-\alpha}\right|^{2} \circ F^{k}(x) \leq e^{n(\eta+\epsilon)} .
$$

We set $\delta(t):=n(t)-\frac{t}{\rho(F)}$. By the Lemma 4.1, $\delta_{2}$ is a bounded function. Since for all $t \in \mathbb{R}$

$$
E(t)=\phi(t) \int_{I_{0}} \frac{\left|f^{\prime}(x)\right|^{2}}{D F^{n(t)}(x)} \prod_{k=0}^{n(t)-1}\left|\left(\frac{\beta(x)+\alpha(x)}{\beta(x)-\alpha(x)}\right) \circ F^{k}(x)\right|^{2} d x,
$$

then, by setting $t_{\epsilon}:=h^{-1}\left(I_{N_{\epsilon}}\right)$, the proof is finished. 


\section{Corollary 4.3.}

(i) Under the hypotheses of Theorem 4.2, 1), we have

$$
\begin{aligned}
& F^{\prime}\left(a_{1}\right)<\left|\left(\frac{\beta+\alpha}{\beta-\alpha}\right)\left(a_{1}\right)\right|^{2} \Longrightarrow E \text { grows exponentially. } \\
& F^{\prime}\left(a_{1}\right)>\left|\left(\frac{\beta+\alpha}{\beta-\alpha}\right)\left(a_{1}\right)\right|^{2} \Longrightarrow E \text { decays exponentially. } \\
& F^{\prime}\left(a_{1}\right)=\left|\left(\frac{\beta+\alpha}{\beta-\alpha}\right)\left(a_{1}\right)\right|^{2} \Longrightarrow E \text { is bounded. }
\end{aligned}
$$

(ii) Under the hypotheses of Theorem 4.3, 2), and we assume furthermore that $s$ is constant and is a strictly positif irrational and that

$$
\int_{0}^{1} \ln \left|\frac{\beta(x)+\alpha(x)}{\beta(x)-\alpha(x)}\right|^{2} d x=: \eta \neq 0 .
$$

Then, for all $\epsilon>0$, there exists a real $t_{\epsilon} \geq 0$ such that for all $t \geq t_{\epsilon}$, we have

$$
\begin{aligned}
& \min \left\{1, \frac{1}{F_{\text {max }}^{\prime}}\left(\frac{\beta+\alpha}{\beta-\alpha}\right)_{\text {min }}^{4}\right\}\left\|f^{\prime}\right\|_{I_{0}}^{2} \exp \left(t \frac{\eta-\epsilon}{2 a}+\delta(t)(\eta-\epsilon)\right) \\
& \leq E(t) \leq \\
& \max \left\{1, \frac{1}{F_{\text {min }}^{\prime}}\left(\frac{\beta+\alpha}{\beta-\alpha}\right)_{\text {max }}^{4}\right\}\left\|f^{\prime}\right\|_{I_{0}}^{2} \exp \left(t \frac{\eta+\epsilon}{2 a}+\delta(t)(\eta+\epsilon)\right) .
\end{aligned}
$$

Proof. The first part of the corollary is easy to prove. For the second one, we have $F=I d+2 a$ which is an irrational translation, and hence the unique invariant measure is the Lebesgue's measure, and $\rho(F)=2 a$.

Now, we consider the problem

$$
\begin{aligned}
& u_{t t}-u_{x x}=0 \quad \text { in } \quad(0, s(t)) \times \mathbb{R}, \\
& \alpha(t) u_{x}(0, t)+\beta(t) u_{t}(0, t)=0, \quad t \in \mathbb{R}, \\
& u_{x}(s(t), t)=0, \quad t \in \mathbb{R}, \\
& u(x, 0)=u_{0}(x), \quad u_{t}(x, 0)=u_{1}(x), \quad x \in(0, s(0)) .
\end{aligned}
$$

Then, we have 
Theorem 4.4. Let $s \in \operatorname{Lip}(\mathbb{R})$ such that $L(s) \in[0,1)$, $s>0$, and $\alpha, \beta \in$ $C^{0}(\mathbb{R})$ such that

$$
\exists v \in \mathbb{R}_{+}^{*}, \quad \forall t \in \mathbb{R}, \quad|\alpha(t) \pm \beta(t)| \geq v .
$$

Let $\left(u_{0}, u_{1}\right) \in H^{1}((0, s(0))) \times L^{2}((0, s(0)))$. Then, there exists a unique weak solution $u$ to problem (4.8)-(4.11) given by

$$
u(x, t)=f(t+x)+g(t-x) \quad \text { a.e. in } \Omega,
$$

$f$ and $g$ are two functions belonging to $H_{\text {loc }}^{1}(\mathbb{R})$.

Furthermore, if $s, \alpha, \beta$ are 1-periodic and $E(0)>0$ then:

1. Assume that $\rho(F)=p$ with $p \in \mathbb{N}^{*}$ and that $F$ has only two periodic points $a_{1}, a_{2}$ in $I_{0}$, one of which $a_{1}$ is hyperbolic and attracting. Assume that $s \in C^{1}(\mathbb{T}), G:=\left|\frac{\beta-\alpha}{\beta+\alpha}\right|^{2} \cdot F^{\prime} \in \operatorname{Lip}(\mathbb{T})$ and $G\left(a_{1}\right)>G\left(a_{2}\right)$. Then,

$$
\forall t \in \mathbb{R}, \quad E(t)=\phi(t)\left\|f^{\prime}\right\|_{I_{n}(t)}^{2}
$$

with $n(t)=\frac{t}{p}+\delta_{1}(t), \delta_{1}$ bounded and $p$-periodic,

$$
\begin{aligned}
& 0<\min \left\{1, \frac{1}{F_{\text {max }}^{\prime}}\left(\frac{\beta+\alpha}{\beta-\alpha}\right)_{\text {min }}^{4}\right\} \leq \\
& \phi \leq \max \left\{1, \frac{1}{F_{\text {min }}^{\prime}}\left(\frac{\beta+\alpha}{\beta-\alpha}\right)_{\text {max }}^{4}\right\}<+\infty
\end{aligned}
$$

and

$$
\left\|f^{\prime}\right\|_{I_{n}(t)}^{2}=\left\|\sqrt{l^{-1}} f^{\prime}\right\|_{I_{0}}^{2} G\left(a_{1}\right)^{n(t)}(1+o(1)) \quad \text { as } \quad t \rightarrow+\infty
$$

with $l(x):=\prod_{k=0}^{+\infty} \frac{G\left(a_{1}\right)}{G \circ F^{k}(x)}, x \in I_{0} ; l^{-1} \in C^{0}\left(I_{0} \backslash\left\{a_{2}\right\}\right), l^{-1}\left(a_{2}\right)=0$.

2. Assume that $\rho(F) \in \mathbb{R} \backslash \mathbb{Q}$ and denote by $\mu$ the unique invariant measure by $\bar{F}$. If the Radon-Nikodym derivative of $\mu$ with respect to Lebesgue's measure $m$ satisfies

$$
0<\lambda_{1} \leq \frac{d \mu}{d m} \leq \lambda_{2}<+\infty \quad \text { for } \quad \lambda_{1}, \lambda_{2} \in \mathbb{R}
$$

and if

$$
\int_{\mathbb{T}} \ln \left|\frac{\beta+\alpha}{\beta-\alpha}\right|^{2} d \mu=: \eta \neq 0,
$$


then for all $\epsilon>0$ there exists a real $t_{\epsilon} \geq 0$ such that for all $t \geq t_{\epsilon}$ :

$$
\begin{gathered}
\min \left\{1, \frac{1}{F_{\text {max }}^{\prime}}\left(\frac{\beta+\alpha}{\beta-\alpha}\right)_{\min }^{4}\right\} \frac{\lambda_{1}\left\|f^{\prime}\right\|_{I_{0}}^{2}}{\lambda_{2}} \exp \left(t \frac{\eta-\epsilon}{\rho(F)}+\delta(t)(\eta-\epsilon)\right) \\
\leq E(t) \leq \\
\max \left\{1, \frac{1}{F_{\text {min }}^{\prime}}\left(\frac{\beta+\alpha}{\beta-\alpha}\right)_{\max }^{4}\right\} \frac{\lambda_{2}\left\|f^{\prime}\right\|_{I_{0}}^{2}}{\lambda_{1}} \exp \left(t \frac{\eta+\epsilon}{\rho(F)}+\delta(t)(\eta+\epsilon)\right)
\end{gathered}
$$

where $\delta$ is a bounded function in $\mathbb{R}$.

Proof. The proof is similar to that of Theorem 4.2. The relations which permit to construct the solution $u(x, t)=f(t+x)+g(t-x)$ a.e. in $\Omega$ are

$$
g^{\prime}=f^{\prime} \circ F, \quad g^{\prime}=\frac{\alpha+\beta}{\alpha-\beta} f^{\prime} \quad \text { a.e. in } \quad \mathbb{R} .
$$

For the energy estimates, we use the fact that

$$
\forall n \in \mathbb{N}^{*}, \quad \int_{I_{n}}\left|f^{\prime}\right|^{2} d m=\int_{I_{0}}\left|f^{\prime}\right|^{2} D F^{n} \prod_{k=0}^{n-1}\left|\frac{\beta+\alpha}{\beta-\alpha}\right|^{2} \circ F^{k} d m .
$$

We, then, apply Lemma 4.1 in the case $G\left(a_{1}\right)>G\left(a_{2}\right)$ to get 1$)$.

For part 2) of the Theorem, the proof is similar since the term $D F^{n}$ does not play any role.

\section{Inhomogeneous Boundary Conditions}

In this section we assume that the Dirichlet boundary conditions are inhomogeneous, more precisely consider the problem:

$$
\begin{aligned}
& u_{t t}-u_{x x}=0, \quad \text { in } \quad(0, s(t)) \times \mathbb{R}, \\
& u(0, t)=\alpha(t), \quad u(s(t), t)=\beta(t), \quad t \in \mathbb{R}, \\
& u(x, 0)=u_{0}(x), \quad u_{t}(x, 0)=u_{1}(x), \quad \text { in } \quad(0, s(0)) .
\end{aligned}
$$

Our aim is to study the asymptotic behavior of the energy defined by

$$
\forall t \in \mathbb{R}, \quad E(t):=\frac{1}{2} \int_{0}^{s(t)}\left(\left|u_{t}(x, t)\right|^{2}+\left|u_{x}(x, t)\right|^{2}\right) d x .
$$


To get an idea how the energy could behave, assume for a while that $s(t):=$ $s_{0}>0, \alpha:=0, \beta(t):=A \sin \frac{2 \pi}{T} t$ with $A \neq 0$ and $T>0$. Then, $u(x, t)=$ $\frac{A}{\sin \frac{2 \pi}{T} a_{0}} \sin \frac{2 \pi}{T} x \sin \frac{2 \pi}{T} t$ is a solution to (5.1)-(5.3). In this particular case, the energy has the form

$$
E(t)=\frac{|A|^{2} \pi^{2}}{T^{2} \sin ^{2} \frac{2 \pi}{T} a_{0}}\left(a_{0}-\frac{1}{2} \sin 2 a_{0} \cos \frac{4 \pi}{T} t\right) .
$$

If $\frac{T}{a_{0}}=\frac{2}{k}, k \in \mathbb{N}^{*}$, then the system has a resonance. If $\frac{T}{a_{0}} \in \mathbb{R} \backslash \mathbb{Q}$, there is no resonance. However, if $\frac{T}{a_{0}}$ is closed to a rational number, then $\sin \frac{2 \pi}{T} a_{0}$ may be arbitrarily small and the energy may become arbitrarily large.

For the existence, we have: ( $F$ and $h$ are defined in section 2$)$.

Theorem 5.1. Let $s \in \operatorname{Lip}(\mathbb{R})$ be such that $L(s) \in[0,1)$, $s>0$, and let $\alpha, \beta \in C^{1}(\mathbb{R})$. For any $\left(u_{0}, u_{1}\right) \in H^{1}((0, s(0))) \times L^{2}((0, s(0)))$ such that $u_{0}(0)=\alpha(0)$ and $u_{0}(s(0))=\beta(0)$, there exists a unique weak solution $u$ to problem (5.1)-(5.3). Furthermore, there exists a function $f \in H_{l o c}^{1}(\mathbb{R})$ such that

$$
u(x, t)=f(t+x)-f(t-x)+\alpha(t-x) \quad \text { a.e. in } \Omega .
$$

Proof. By proposition 2.2, the weak solution, if it exists, is given by $u(x, t)=$ $f(t+x)+g(t-x)$ a.e. in $\Omega$. The boundary conditions imply that $g=-f+\alpha$ and

$$
\begin{aligned}
f \circ F & =f+\gamma \\
\gamma & :=\beta \circ h^{-1}-\alpha .
\end{aligned}
$$

Hence, we can construct a solution with the help of the relations

$$
\forall n \in \mathbb{N}^{*}, \quad f \circ F^{n}=f+\sum_{k=0}^{n-1} \gamma \circ F^{k}, f \circ F^{-n}=f-\sum_{k=0}^{n-1} \gamma \circ F^{-k}
$$

and the value of $f$ on the interval $I_{0}$. Indeed, since $f(0)+g(0)=\alpha(0), g(-x)=$ $g(0)+\frac{1}{2}\left(u_{0}(x)-\alpha(0)-\int_{0}^{x} u_{1} d m\right)=-f(-x)+\alpha(-x)$, we deduce that

$$
\begin{aligned}
\forall x \in[0, s(0)], f(x) & =f(0)+\frac{1}{2}\left(u_{0}(x)-\alpha(0)+\int_{0}^{x} u_{1}(y) d y\right), \\
f(-x) & =f(0)+\frac{1}{2}\left(-u_{0}(x)+2 \alpha(-x)-\alpha(0)+\int_{0}^{x} u_{1}(y) d y\right)
\end{aligned}
$$


with $f(0)$ an arbitrary constant. We have

$$
\begin{aligned}
f\left(s(0)_{+}\right) & =f \circ F(-s(0))=f(-s(0))+\gamma(-s(0)) \\
& =f(0)+\frac{1}{2}\left(-\alpha(0)+u_{0}(s(0))+\int_{0}^{s(0)} u_{1} d m\right),
\end{aligned}
$$

and hence $f(s(0))-f(-s(0))=\gamma(-s(0))$. Thus, we can construct a function $f \in H_{l o c}^{1}$ on $\mathbb{R}$.

We present now a useful formula for the energy expression. We know that

$$
\forall t \in \mathbb{R}, \quad E(t)=\int_{h(t)}^{t}\left|f^{\prime}(x)-\alpha^{\prime}(x)\right|^{2} d x+\int_{t}^{k(t)}\left|f^{\prime}(x)\right|^{2} d x .
$$

Consider the sequence of integers defined by

$$
t_{n}:=h^{-1}\left(x_{n-1}\right)=h^{-1} \circ F^{n}(-s(0)), \quad n \in \mathbb{N} .
$$

Then, for all $n \in \mathbb{N}$ :

$$
E\left(t_{n}\right)=\int_{x_{n-1}}^{t_{n}}\left|f^{\prime}(x)-\alpha^{\prime}(x)\right|^{2} d x+\int_{t_{n}}^{x_{n}}\left|f^{\prime}(x)\right|^{2} d x .
$$

By the variable change $y:=F^{-n}(x)$, we get for all $n \in \mathbb{N}^{*}$

$$
\begin{aligned}
E\left(t_{n}\right)= & \int_{-s(0)}^{F^{-n}\left(t_{n}\right)}\left|f^{\prime}(x) \circ F^{n}(y)-\alpha^{\prime} \circ F^{n}(y)\right|^{2} D F^{n}(y) d y+ \\
& +\int_{F^{-n}\left(t_{n}\right)}^{s(0)}\left|f^{\prime} \circ F^{n}(y)\right|^{2} D F^{n}(y) d y \\
= & \int_{-s(0)}^{F^{-n}\left(t_{n}\right)} \frac{\left|f^{\prime}+\sum_{k=0}^{n-1} D F^{k} \gamma^{\prime} \circ F^{k}-D F^{n} \alpha^{\prime} \circ F^{n}\right|^{2}}{D F^{n}} d m+ \\
& +\int_{F^{-n}\left(t_{n}\right)}^{s(0)} \frac{\left|f^{\prime}+\sum_{k=0}^{n-1} D F^{k} \gamma^{\prime} \circ F^{k}\right|^{2}}{D F^{n}} d m .
\end{aligned}
$$

We consider the simplified case where $s$ is a positive constant (hence $F=$ $I d+2 s)$. Then, by (5.5), $\gamma(x)=\beta(x+s)-\alpha(x)$ and if we set

$$
\eta_{p, q}:=\sum_{k=0}^{q-1} \gamma^{\prime}\left(x+k \frac{p}{q}\right)=\sum_{k=0}^{q-1}\left(\beta^{\prime}\left(x+k \frac{p}{q}+s\right)-\alpha^{\prime}\left(x+k \frac{p}{q}\right)\right), x \in \mathbb{R},
$$


$p$ and $q$ are two positive integers, the formula (5.6) now becomes

$$
\begin{gathered}
\forall n \in \mathbb{N}^{*}, E(2 s n)=\int_{-s}^{0}\left|f^{\prime}(x)+\sum_{k=0}^{n-1} \gamma^{\prime}(x+2 k s)-\alpha^{\prime}(x+2 s n)\right|^{2} d x+ \\
+\int_{0}^{s}\left|f^{\prime}(x)+\sum_{k=0}^{n-1} \gamma^{\prime}(x+2 k s)\right|^{2} d x
\end{gathered}
$$

If, furthermore, $s:=\frac{p}{2 q}\left(p, q \in \mathbb{N}^{*}\right)$ and $\alpha^{\prime}, \beta^{\prime} \in C^{0}(\mathbb{T})$, then if we set $n:=m q, m \in \mathbb{N}^{*}$, the relation (5.7) becomes

$$
\begin{aligned}
\forall m \in \mathbb{N}^{*}, \quad E(m p)=\int_{-\frac{p}{2 q}}^{0} \mid & f^{\prime}(x)+\eta_{p, q}(x) m-\left.\alpha^{\prime}(x+m p)\right|^{2} d x+ \\
& +m^{2} \int_{0}^{\frac{p}{2 q}}\left|\frac{f^{\prime}(x)}{m}+\eta_{p, q}(x)\right|^{2} d x .
\end{aligned}
$$

Before the statement and proof of the main Theorem of this section, we recall some definitions.

\section{Definition 5.2.}

1. The Sobolev space $H^{m}(\mathbb{T})$ can be defined as

$$
H^{m}(\mathbb{T}):=\left\{f \in L^{2}(\mathbb{T}) ; \quad \sum_{n \in \mathbb{Z}} n^{2 m}\left|f_{n}\right|^{2}<+\infty\right\} .
$$

For every $f \in L^{2}(\mathbb{T})$ we have

$$
f(x)=\sum_{n \in \mathbb{Z}} f_{n} e^{2 \pi i n x} .
$$

2. Let $\lambda \in \mathbb{R}_{+}^{*}$. We say that $\alpha \in \mathcal{I}_{\lambda}$ if there exists a constant $c>0$ such that

$$
\forall \frac{p}{q} \in \mathbb{Q}, \quad\left|\alpha-\frac{p}{q}\right| \leq \frac{c}{q^{2+\lambda}} .
$$

3. Let $\beta \in \mathbb{R}_{+}$. Then $\alpha \in \mathbb{R} \backslash \mathbb{Q}$ is said a $\beta$-diophantian number if there exists $c \in \mathbb{R}_{+}^{*}$ such that

$$
\forall \frac{p}{q} \in \mathbb{Q}, \quad\left|\alpha-\frac{p}{q}\right| \geq \frac{c}{q^{2+\beta}} .
$$


Theorem 5.3. Assume that $s$ is constant and positive,

$$
\left(u_{0}, u_{1}\right) \in H^{1}((0, s(0))) \times L^{2}((0, s(0))) \quad \text { and that } \alpha, \beta \in C^{1}(\mathbb{R})
$$

with $\alpha(0)=u_{0}(0), \beta(0)=u_{0}(s(0))$.

1) If $s=\frac{p}{2 q} \in \mathbb{Q}^{*}\left(p, q \in \mathbb{N}^{*}\right), \alpha^{\prime}, \beta^{\prime} \in C^{0}(\mathbb{T})$, then

$$
\forall n \in \mathbb{N}^{*}, \quad E(n p)=A n^{2}+B n+C
$$

with

$$
\begin{aligned}
A & :=\int_{-s}^{s}\left|\eta_{p, q}(x)\right|^{2} d x, \\
B & :=2\left(\int_{-s}^{s} \eta_{p, q}(x) f^{\prime}(x) d x-\int_{-s}^{0} \eta_{p, q}(x) \alpha^{\prime}(x+n p) d x\right), \\
C & :=\int_{-s}^{s}\left|f^{\prime}(x)\right|^{2} d x+\int_{-s}^{0}\left(\alpha^{\prime}(x+n p)^{2}-2 \alpha^{\prime}(x+n p) f^{\prime}(x)\right) d x .
\end{aligned}
$$

2) If $s \in \mathbb{R} \backslash \mathbb{Q}$, we assume that $\alpha, \beta \in H^{1}(\mathbb{T})$, then we have:

a) If $s>1,\left|\gamma_{n}^{\prime}\right| \geq c|n|^{-\eta}, c>0, \eta \in\left(\frac{1}{2}, 1\right)$, and if $u_{0}^{\prime}+u_{1}=0$ a.e. on $(0, s)$, then there exists $d>0$ such that

$$
\forall n \in \mathbb{N}, \quad E(n s)>d n^{2(1-\eta)} .
$$

b) If $s>1, s \in \mathcal{I}_{\lambda}, \lambda \in \mathbb{R}_{+}^{*}$, and if $u_{0}^{\prime}+u_{1}=0$ a.e. on $(0, s)$, then

$$
\limsup _{t \rightarrow+\infty} E(t)=+\infty \text {. }
$$

If $s \in \mathbb{R} \backslash \mathbb{Q}, \alpha, \beta \in H^{4}(\mathbb{T})$, then

$\liminf _{t \rightarrow+\infty} E(t) \leq 2\left\|f^{\prime}\right\|_{(-s, s)}^{2}+4(\lfloor s\rfloor+1) \operatorname{Var}\left(\gamma^{\prime}\right)^{2}+2 s\left\|\alpha^{\prime}\right\|_{L^{\infty}(\mathbb{T})}^{2}<+\infty$.

c) If $s$ is $\lambda$-diophantian with $\lambda \in[0,1]$ and $\alpha, \beta \in H^{4}(\mathbb{T})$, then for all $t \in \mathbb{R}_{+}$:

$$
\begin{gathered}
E(t) \leq \frac{2(\lfloor s\rfloor+1)}{\pi^{4} c^{2}}\left\|D^{3} \gamma\right\|_{\mathbb{T}}^{2} \\
+16\left\|f^{\prime}\right\|_{(-s, s)}^{2}+16 s\left(2\left\|\alpha^{\prime}\right\|_{L^{\infty}(\mathbb{T})}^{2}+\left\|\beta^{\prime}\right\|_{L^{\infty}(\mathbb{T})}^{2}\right)<+\infty
\end{gathered}
$$

where $c$ is the constant appearing in the definition af a diophantine number. 


\section{Proof.}

1) The relation (5.9) follows easily from (5.8).

2) a) Since $f^{\prime}=0$ a.e. on $(0, s(0))$, then by (5.7) we have for all $n \in \mathbb{N}^{*}$

$$
E(2 s n) \geq \int_{0}^{1}\left|\sum_{k=0}^{n-1} \gamma^{\prime}(x+2 k s)\right|^{2} d x .
$$

Since $\alpha, \beta \in H^{1}(\mathbb{T})$, we have $\gamma^{\prime} \in L^{2}(\mathbb{T})$, and also $\int_{0}^{1} \gamma^{\prime} d m=0$.

If $\left|\gamma_{n}^{\prime}\right| \geq c|n|^{-\eta}, \eta \in\left(\frac{1}{2}, 1\right)$, then thanks to [10, Th. 1.3] we deduce that

$$
\forall n \in \mathbb{N}^{*}, \quad E(2 s n) \geq d n^{2(1-\eta)},
$$

for $d \in \mathbb{R}_{+}^{*}$.

2) b) $\gamma^{\prime} \in L^{2}(\mathbb{T})$ and by (5.7) we have for all $n \in \mathbb{N}^{*}$ and thanks to [10, Th. 1.1]

$$
\begin{aligned}
\frac{1}{n} \sum_{j=1}^{n} E(2 s j) & \geq \frac{1}{n} \int_{0}^{1} \sum_{j=1}^{n}\left|\sum_{k=0}^{j-1} \gamma^{\prime}(x+2 s k)\right|^{2} d x \\
& \geq \frac{1}{n^{2}} \int_{0}^{1}\left|\sum_{j=1}^{n} \sum_{k=0}^{j-1} \gamma^{\prime}(x+2 s k)\right|^{2} d x \\
& \geq c n^{2\left(1-\frac{\eta}{1+\lambda}\right)\left(\frac{1+\lambda}{1+\lambda+\eta \lambda}\right)},
\end{aligned}
$$

where $c$ is a positive constant depending only on $\lambda$ and

$$
\left(1-\frac{\eta}{1+\lambda}\right)\left(\frac{1+\lambda}{1+\lambda+\eta \lambda}\right)>0 \text {. }
$$

Hence

$$
\lim _{n \rightarrow+\infty} \frac{1}{n} \sum_{j=1}^{n} E(2 s j)=+\infty .
$$

Thus, we have proved (5.10). 
If $m \geq 3$, then $\gamma^{\prime} \in C^{2}(\mathbb{T})$. Since $s$ is irrational we can hence apply the Denjoy - Koksma inequality (Cf. [17, Th. VI.3.1]):

$$
\forall x \in[0,1], \quad\left|\sum_{k=0}^{q_{n}-1} \gamma^{\prime}(x+2 s k)\right| \leq \operatorname{Var}\left(\gamma^{\prime}\right),
$$

where $\operatorname{Var}\left(\gamma^{\prime}\right)$ is the total variation of $\gamma^{\prime}$.

Hence 2) b) is now proved, because (5.7) implies

$\forall n \in \mathbb{N}^{*}, E\left(2 s q_{n}\right) \leq 2\left\|f^{\prime}\right\|_{(-s, s)}^{2}+4(\lfloor s\rfloor+1) \operatorname{Var}\left(\gamma^{\prime}\right)^{2}+2 s\left\|\alpha^{\prime}\right\|_{L^{\infty}(\mathbb{T})}^{2}<+\infty$.

2) c) We use a result due to Herman (Cf. [35]): if $\lambda \in[0,1]$ and $\gamma^{\prime} \in$ $H^{k}(\mathbb{T}), k \geq 2$, then there exists a unique function $\delta \in H^{k}(\mathbb{T})$ such that $\delta \circ R_{2 s}-\delta=\gamma^{\prime}$. Furthermore,

$$
\|\delta\|_{\mathbb{T}}^{2} \leq \frac{1}{(2 \pi)^{4} c^{2}}\left\|D^{3} \gamma\right\|_{\mathbb{T}}^{2}
$$

where $c$ is the constant appearing in the definition of a diophantine number.

Hence, $\delta \circ R_{2 s(n-1)}-\delta=\sum_{k=0}^{n-1} \gamma^{\prime} \circ R_{2 k s}$, and

$$
\sup _{n \in \mathbb{N}}\left\|\sum_{k=0}^{n-1} \gamma^{\prime} \circ R_{2 k s}\right\|_{\mathbb{T}}^{2} \leq 2\|\delta\|_{\mathbb{T}}^{2}<+\infty .
$$

Hence, thanks to (5.7), $\forall n \in \mathbb{N}^{*}$

$$
\begin{aligned}
E(2 s n) \leq & 2 \int_{-s}^{s}\left|f^{\prime}(x)\right|^{2} d x+2 \int_{-s}^{s}\left|\sum_{k=0}^{n-1} \gamma^{\prime}(x+2 k s)\right|^{2} d x+ \\
& +2 \int_{-s}^{0}\left|\alpha^{\prime}(x+2 s n)\right|^{2} d x \\
\leq & 2\left\|f^{\prime}\right\|_{(-s, s)}^{2}+2(\lfloor s\rfloor+1) \frac{2}{(2 \pi)^{4} c^{2}}\left\|D^{3} \gamma\right\|_{\mathbb{T}}^{2}+2 s\left\|\alpha^{\prime}\right\|_{L^{\infty}(\mathbb{T})}^{2} .
\end{aligned}
$$


Let $t \in \mathbb{R}_{+}$, then there exists a unique $n \in \mathbb{N}$ such that $h(t) \in I_{n}=[-s+$ $2 n s, s+2 n s]=\left[x_{n-1}, x_{n}\right)$. Assume that $t>x_{n}$, then

$$
\begin{aligned}
E(t)= & \int_{h(t)}^{x_{n}}\left|f^{\prime}-\alpha^{\prime}\right|^{2} d m+\int_{x_{n}}^{t}\left|f^{\prime}-\alpha^{\prime}\right|^{2} d m+\int_{t}^{k(t)}\left|f^{\prime}\right|^{2} d m \\
= & \int_{h(t)}^{x_{n}}\left|f^{\prime}-\alpha^{\prime}\right|^{2} d m+\int_{x_{n-1}}^{F^{-1}(t)}\left|f^{\prime}+\gamma^{\prime}-\alpha^{\prime} \circ F\right|^{2} d m+ \\
& \quad+\int_{F^{-1}(t)}^{h(t)}\left|f^{\prime}+\gamma^{\prime}\right|^{2} d m \\
\leq & 2 \int_{I_{n}}\left|f^{\prime}-\alpha^{\prime}\right|^{2} d m+4 s\left(2\left\|\beta^{\prime}\right\|_{L^{\infty}(\mathbb{T})}^{2}+\left\|\alpha^{\prime}\right\|_{L^{\infty}(\mathbb{T})}^{2}\right) \\
\leq & 4\left(\int_{x_{n-1}}^{x_{n-1}+s}\left|f^{\prime}-\alpha^{\prime}\right|^{2} d m+\int_{x_{n-1}+s}^{x_{n}}\left|f^{\prime}\right|^{2} d m\right)+ \\
& \quad+8 s\left(\left\|\beta^{\prime}\right\|_{L^{\infty}(\mathbb{T})}^{2}+\left\|\alpha^{\prime}\right\|_{L^{\infty}(\mathbb{T})}^{2}\right) \\
= & 4 E(2 n s)+8 s\left(\left\|\beta^{\prime}\right\|_{L^{\infty}(\mathbb{T})}^{2}+\left\|\alpha^{\prime}\right\|_{L^{\infty}(\mathbb{T})}^{2}\right) \\
\leq & \frac{\lfloor a\rfloor+1}{\pi^{4} c^{2}}\left\|D^{3} \gamma\right\|_{\mathbb{T}}^{2}+8 s\left(\left\|\beta^{\prime}\right\|_{L^{\infty}(\mathbb{T})}^{2}+2\left\|\alpha^{\prime}\right\|_{L^{\infty}(\mathbb{T})}^{2}\right)+8\left\|f^{\prime}\right\|_{(-s, s)}^{2} .
\end{aligned}
$$

If $t \leq x_{n}$, we similarly obtain

$$
\begin{aligned}
E(t) & =\int_{h(t)}^{t}\left|f^{\prime}-\alpha^{\prime}\right|^{2} d m+\int_{t}^{x_{n}}\left|f^{\prime}\right|^{2} d m+\int_{x_{n}}^{k(t)}\left|f^{\prime}\right|^{2} d m \\
& =\int_{h(t)}^{t}\left|f^{\prime}-\alpha^{\prime}\right|^{2} d m+\int_{F^{-1}(t)}^{x_{n-1}}\left|f^{\prime}+\gamma^{\prime}\right|^{2} d m+\int_{x_{n-1}}^{h(t)}\left|f^{\prime}+\gamma^{\prime}\right|^{2} d m \\
& \leq 2 \int_{x_{n-1}}^{t}\left|f^{\prime}-\alpha^{\prime}\right|^{2} d m+2 \int_{F^{-1}(t)}^{x_{n-1}}\left|f^{\prime}-\alpha^{\prime}\right|^{2} d m+8 s\left\|\beta^{\prime}\right\|_{L^{\infty}(\mathbb{T})}^{2} \\
& =2 \int_{x_{n-2}}^{F^{-1}(t)}\left|f^{\prime}+\gamma^{\prime}-\alpha^{\prime} \circ F\right|^{2} d m+2 \int_{F^{-1}(t)}^{x_{n-1}}\left|f^{\prime}-\alpha^{\prime}\right|^{2} d m+8 s\left\|\beta^{\prime}\right\|_{L^{\infty}(\mathbb{T})}^{2} \\
& \leq 4 \int_{I_{n-2}}\left|f^{\prime}-\alpha^{\prime}\right|^{2} d m+8 s\left\|\alpha^{\prime}\right\|_{L^{\infty}(\mathbb{T})}^{2}+16 s\left\|\beta^{\prime}\right\|_{L^{\infty}(\mathbb{T})}^{2} \\
& \leq 8 E(2(n-1) s)+16 s\left\|\alpha^{\prime}\right\|_{L^{\infty}(\mathbb{T})}^{2}+16 s\left\|\beta^{\prime}\right\|_{L^{\infty}(\mathbb{T})}^{2} \\
& \leq \frac{2(\lfloor s\rfloor+1)}{\pi^{4} c^{2}}\left\|D^{3} \gamma\right\|_{\mathbb{T}}^{2}+16 s\left(\left\|\beta^{\prime}\right\|_{L^{\infty}(\mathbb{T})}^{2}+2\left\|\alpha^{\prime}\right\|_{L^{\infty}(\mathbb{T})}^{2}\right)+16\left\|f^{\prime}\right\|_{(-s, s)}^{2} .
\end{aligned}
$$

Hence, the energy is uniformly bounded. 
Remark 5.4. If we consider the problem

$$
\begin{gathered}
u_{t t}-u_{x x}=p(t) \quad \text { in } \quad(0, s(t)) \times \mathbb{R}, \\
u(0, t)=u(s(t), t)=0, \quad t \in \mathbb{R}, \\
u(x, 0)=u_{0}(x), \quad u_{t}(x, 0)=u_{1}(x) \quad \text { in }(0, s(0)),
\end{gathered}
$$

with $s \in C^{1}(\mathbb{R}), s>0,\left|s^{\prime}\right|<1$. The energy is defined for all $t \in \mathbb{R}$ by

$$
E(t):=\frac{1}{2} \int_{0}^{s(t)}\left(\left|u_{t}(x, t)\right|^{2}+\left|u_{x}(x, t)\right|^{2}-p(t) u(x, t)\right) d x .
$$

Assume that $p \in L_{l o c}^{1}(\mathbb{R})$ and if we set

$$
P(t):=\int_{0}^{t} \int_{0}^{\xi} p(\theta) d \theta d \xi
$$

then $P \in C^{1}(\mathbb{R})$. The variable change $v:=u-P$ yields the new problem

$$
\begin{gathered}
v_{t t}-v_{x x}=0 \quad \text { in }(0, s(t)) \times \mathbb{R}, \\
v(0, t)=-P(t), \quad v(s(t), t)=-P(s(t)), \quad t \in \mathbb{R}, \\
v(x, 0)=u_{0}(x), \quad v_{t}(x, 0)=u_{1}(x) \text { in }(0, s(0)) .
\end{gathered}
$$

If we set $\alpha(t):=-P(t)$ and $\beta(t):=-P(s(t))$ and by imposing appropriate conditions on $p$ and $s$, we can apply the results of Theorem 5.3 to the above problem.

\section{Stabilization Results}

When the boundary $s$ is no longer periodic or monotone, it seems difficult to estimate the energy. Our aim in this section is to establish some results on the asymptotic behavior of the energy when

$$
\forall t \in \mathbb{R}, \quad s(t)=s_{1}(t)+s_{\infty}>0,
$$

where $s_{1} \in C^{1}(\mathbb{R}) \cap L^{\infty}(\mathbb{R}),\left|s_{1}^{\prime}\right|<1$ and $s_{\infty} \in \mathbb{R}_{+}^{*}, \lim _{|t| \rightarrow+\infty} s_{1}(t)=0$. We assume that this function $s$ is the moving boundary in the Dirichlet case

$$
\begin{aligned}
& u_{t t}-u_{x x}=0 \quad \text { in } \quad(0, s(t)) \times \mathbb{R}, \\
& u(0, t)=0, u(s(t), t)=0, \quad t \in \mathbb{R}, \\
& u(x, 0)=u_{0}(x), \quad u_{t}(x, 0)=u_{1}(x), \quad \text { in }(0, s(0)) .
\end{aligned}
$$


Problem (6.1)-(6.3) is a stabilization problem since asymptotically the boundary is fixed and for intermediate times it is perturbed by the function $s_{1}$. We will prove that the energy may be bounded or not according to the behavior of $s_{1}$.

We define

$$
\phi:=2 s_{1} \circ\left(I d-s_{1}-s_{\infty}\right)^{-1}+2 s_{\infty}, \quad F:=I d+\phi .
$$

It is easy to check that

$$
F \in \operatorname{Diff}^{1}(\mathbb{R}), \quad \text { and } \quad s=\left(\frac{\phi}{2}\right) \circ\left(\frac{\phi}{2}+I d\right)^{-1} .
$$

The existence results are similar to those presented in Theorems 4.2 and 4.4. So we omit here the details.

The main result of this section is:

Theorem 6.1. Assume that $\left(u_{0}, u_{1}\right) \in H_{0}^{1}((0, s(0))) \times L^{2}((0, s(0))), E(0)>$ $0, s:=s_{1}+s_{\infty}$ with $s_{1} \in C^{1}(\mathbb{R}) \cap L^{\infty}(\mathbb{R}),\left|s_{1}^{\prime}\right|<1$ and $s_{\infty} \in \mathbb{R}_{+}^{*}$.

1) Let $c_{1}, x_{1} \in \mathbb{R}_{+}^{*}, \alpha_{ \pm}>1$ be such that

$$
\forall x \geq x_{1}, \quad\left|\phi^{\prime}(x)\right| \leq \frac{c_{1}}{x^{\alpha_{+}}} \quad \text { and } \quad \forall x \leq-x_{1}, \quad\left|\phi^{\prime}(x)\right| \leq \frac{c_{1}}{|x|^{\alpha_{-}}} .
$$

Then, there exist two positive constants $\gamma_{-}$and $\gamma_{+}$such that

$$
\forall t \in \mathbb{R}, \quad \gamma_{-} \leq E(t) \leq \gamma_{+} .
$$

2) Let $c_{2}, x_{2} \in \mathbb{R}_{+}^{*}, \delta_{ \pm}>\frac{1}{2}$ be such that

$$
\forall x \geq x_{2}, \quad\left|\phi^{\prime}(x)\right| \leq \frac{c_{2}}{x^{\delta_{+}}} \quad \text { and } \quad \forall x \leq-x_{2}, \quad\left|\phi^{\prime}(x)\right| \leq \frac{c_{2}}{|x|^{\delta_{-}}}
$$

and $\sum_{k=0}^{+\infty} \phi^{\prime} \circ F^{k}(x)=-\infty$ for a set $A$ of elements $x \in I_{0}$, the Lebesgue measure of $A$ being positive. If $u_{0}^{\prime}+u_{1} \neq 0$ on $A \cap(0, s(0))$ or $u_{0}^{\prime}-u_{1} \neq 0$ on $A \cap(-s(0), 0)$, then we have

$$
\lim _{|t| \rightarrow+\infty} E(t)=+\infty
$$


Proof. Easily we have

$$
\left\|f^{\prime}\right\|_{(h(t), k(t))}^{2}=\left\|f^{\prime}\right\|_{\left(h(t), x_{n}\right)}^{2}+\left\|\left(F^{\prime}\right)^{-1 / 2} f^{\prime}\right\|_{\left(x_{n-1}, h(t)\right)}^{2} .
$$

By the variable change $x:=F^{n}(y)$, we get for all $n \in \mathbb{Z}$ :

$$
\left\|f^{\prime}\right\|_{I_{n}}^{2}=\left\|\frac{f^{\prime}}{\sqrt{D F^{n}}}\right\|_{I_{0}}^{2}>0 \text {. }
$$

For all $t \in \mathbb{R}$ we define

$$
\alpha(t):=\frac{\left\|f^{\prime}\right\|_{(h(t), k(t))}^{2}}{\left\|f^{\prime}\right\|_{I_{n}(t)}^{2}}
$$

and hence

$$
\forall t \in \mathbb{R}, \quad E(t)=\alpha(t)\left\|\frac{f^{\prime}}{\sqrt{D F^{n(t)}}}\right\|_{I_{0}}^{2} .
$$

1) We will prove that $D F^{n}$ is bounded and converges uniformly to zero on a set of nonzero measure. Remark that

$$
D F^{n}=\prod_{k=0}^{n-1} F^{\prime} \circ F^{k}=\prod_{k=0}^{n-1}\left(1+\phi^{\prime} \circ F^{k}\right) .
$$

We know that if the serie $\sum_{k=0}^{+\infty}\left|\phi^{\prime} \circ F^{k}(x)\right|^{2}$ is convergente, then the convergence of $D F^{n}$ is equivalent to the convergence of $\sum_{k=0}^{+\infty} \phi^{\prime} \circ F^{k}(x)$.

For all $x \geq x_{1}$, we have

$$
\sum_{k=0}^{+\infty}\left|\phi^{\prime} \circ F^{k}(x)\right|^{2} \leq \sum_{k=0}^{+\infty} \frac{c_{1}^{2}}{\left|F^{k}(x)\right|^{2 \alpha_{+}}} .
$$

But, $x+\phi_{\min } \leq F(x) \leq x+\phi_{\max }$ for all $x \in \mathbb{R}$ and hence

$$
\forall x \geq x_{1}, \quad \sum_{k=0}^{+\infty}\left|\phi^{\prime} \circ F^{k}(x)\right|^{2} \leq c_{1}^{2} \sum_{k=0}^{+\infty} \frac{1}{\left|x+k \phi_{\min }\right|^{2 \alpha_{+}}}<+\infty .
$$

Similarly, for all $x \geq x_{1}, \sum_{k=0}^{+\infty}\left|\phi^{\prime} \circ F^{k}(x)\right|<+\infty$ because $\alpha_{ \pm}>1$. These results hold true in the case $x \leq-x_{1}$. Thus, $D F^{n}$ is uniformly bounded, and then $E$ is bounded.

2) The hypotheses ensure that the serie $\sum_{k=0}^{+\infty}\left|\phi^{\prime} \circ F^{k}\right|^{2}$ is convergent and that the product $D F^{n}=\exp \left(\sum_{k=0}^{n-1} \ln \left(1+\phi^{\prime} \circ F^{k}\right)\right)$ is divergent and of limit zero. This divergence is uniforme and hence $E$ is divergent. 


\section{References}

[1] M. Aassila, On some hyperbolic-parabolic equations in domains with moving boundary, J. Inequalities and Applications, to appear.

[2] N. Balasz, On the solution of the wave equation with moving boundaries, J. Math. Anal. Appl. 3 (1961), 472-484.

[3] G. Calucci, Casimir effect for moving bodies, J. Phys. 25 (1992), 3873-3882.

[4] S. N. Chow, K. Lu and J. Mallet-Paret, Floquet theory for parabolic equations: the time periodic case, J. Diff. Equations 109 (1994), 147-200.

[5] J. Cooper, Asymptotic behavior for the vibrating string with a moving boundary, J. Math. Anal. Appl. 174 (1993), 67-87.

[6] J. Cooper and H. Koch, The spectrum of a hyperbolic evolution operator, J. Funct. Analysis 133 (1995), 301-328.

[7] J. Cooper, Long time behavior and energy growth for electromagnetic waves reflected by a moving boundary, IEEE Transac. Ant. Prop. 41 (1993), 13651370 .

[8] J. Cooper, G. Perla Menzala and W. A. Strauss, On the scattering frequencies of time-dependent potentials, Math. Methods in the Appl. Sci. 8 (1986), 576-584.

[9] I. P. Cornfeld, S. V. Fomin and Ya. G. Sinai, Ergodic Theory, Springer-Verlag, New York, 1982.

[10] S. De Bièvre and G. Forni, On the growth of averaged Weyl sums for rigid rotations, Studia Math. 130 (1998), 199-212.

[11] V. V. Dodonov, A. B. Klimov and D. E. Nikonov, Quantum particle in a box with moving walls, J. Math. Phys. 34 (1993), 3391-3404.

[12] V. V. Dodonov, A. B. Klimov and D. E. Nikonov, Quantum phenomena in resonators with moving walls, J. Math. Phys. 34 (1993), 2742-2756.

[13] E. Fermi, On the origin of the cosmic radiation, Phys. Rev. 75 (1949), 11691174.

[14] J. L. Ferrel and L. A. Medeiros, Kirchhoff-Carrier elastic strings in noncylindrical domains, Portugaliae Math. 56 (1999), 465-500.

[15] H. Furstenberg, Strict ergodicity and transformations of the torus, American J. of Math. 83 (1961), 573-601.

[16] N. Gonzalez, An example of pure stability for the wave equation with moving boundary, J. Math. Anal. Appl. 228 (1998), 51-59.

[17] M. Herman, Sur la conjugaison différentiable des difféomorphismes du cercle à des rotations, Publ. Math. Inst. Hautes Etudes Sci. 49 (1979), 5-234. 
[18] H. Johnston and S. Sarkar, A re-examination of the quantum field theory of optical cavities with moving mirrors, J. Phys. 29 (1996), 1741-1746.

[19] G. Karner, On the quantum Fermi accelerator and its relevance to "Quantum Chaos", Lett. Math. Phys. 17 (1989), 180-187.

[20] A. Katok and B. Hasselblatt, Introduction to the Modern Theory of Dynamical Systems, Cambridge University Press, Cambridge, 1995.

[21] V. Komornik and E. Zuazua, A direct method for the boundary stabilization of the wave equation, J. Math. Pures et Appl. 69 (1990), 33-54.

[22] A. Kufner, O. John and S. Fučíc, Function Spaces, Noordhoff International Publishing, Leyden, Academia, Prague, 1977.

[23] J. -L. Lions, Quelques Méthodes de Résolution des Problèmes aux Limites Non Linéaires, Dunod, Paris, 1969.

[24] G. T. Moore, Quantum field theory of the electromagnetic field in a variable length one dimensional cavity, J. Math. Phys. 11 (1970), 2679-2691.

[25] M. Nakao, Decay of classical solutions of a semilinear wave equation, Math. Rep. 7 (1977), 30-40.

[26] M. Nakao and T. Narazaki, Existence and decay of solutions of some nonlinear wave equations in noncylindrical domains, Math. Rep. 11 (1978), 117-125.

[27] G. Perla Menzala, On classical solution of a quasilinear hyperbolic equation, Nonlinear Anal. 3 (1978), 613-627.

[28] L. D. Pustyl'nikov, On Ulam's problem, Theoret. Math. Phys. 57 (1983), 10351038 .

[29] L. D. Pustyl'nikov, Poincaré models, rigorous justification and the second element of thermodynamics on the basis of mechanics, and the Fermi accelerator mechanism, Russian Math. Surveys, 50 (1995), 145-189.

[30] L. D. Pustyl'nikov, A new mechanism of particle acceleration and a relavistic analogue of the Fermi-Ulam model, Theoret. Math. Phys. 77 (1988), 1110-1115.

[31] L. D. Pustyl'nikov, A new mechanism of particle acceleration and rotation numbers, Theoret. Math. Phys. 82 (1990), 180-187.

[32] M. Queffélec, Substitution Dynamical Systems. Spectral Analysis, LNM 1294, Springer-Verlag, New York, 1987.

[33] S. Ulam, One some statistical properties of dynamical systems, in Proceedings of the 4th Berkeley Symposium in Mathematical and Statistical Problems, Univ. of California Press, Berkeley, 3 (1961), 315-320.

[34] P. Walters, Ergodic Theory. Introductory Lectures, LNM 458, Springer-Verlag, New York, 1975. 
[35] J. -C. Yoccoz, An Introduction to Small Divisors Problems. From Number Theory to Physics, Les Houches 1989, 659-679, Springer-Verlag, Berlin, 1992.

\section{Mohammed Aassila}

Institut de Mathematiques

Universite de Fribourg

Perolles

CH-1700 Fribourg

SWITZERLAND

E-mail: mohammed_aassila@yahoo.com 